\title{
Neutralizing IFNL3 Autoantibodies in Severe COVID-19 Identified Using Molecular Indexing of Proteins by Self-Assembly
}

Joel J. Credle ${ }^{1} \uparrow$, Jonathan Gunn ${ }^{1} \uparrow$, Puwanat Sangkhapreecha ${ }^{1}$, Daniel R. Monaco ${ }^{1}$, Xuwen Alice

Zheng ${ }^{1}$, Hung-Ji Tsai ${ }^{2}$, Azaan Wilbon ${ }^{1}$, William R. Morgenlander ${ }^{1}$, Yi Dong ${ }^{3}$, Sahana

Jayaraman $^{1}$, Lorenzo Tosi ${ }^{4}$, Biju Parekkadan ${ }^{4}$, Alan N. Baer ${ }^{5}$, Mario Roederer ${ }^{6}$, Evan M. Bloch ${ }^{7}$, Aaron A. R. Tobian ${ }^{7}$, Israel Zyskind ${ }^{8}$, Jonathan I. Silverberg9, Avi Z. Rosenberg ${ }^{10}$, Andrea L. Cox $^{11}$, Tom Lloyd ${ }^{12}$, Andrew L. Mammen ${ }^{13}$, H. Benjamin Larman ${ }^{1 *}$

10
${ }^{1}$ Institute for Cell Engineering, Division of Immunology, Department of Pathology, Johns Hopkins University School of Medicine; Baltimore, MD, USA.

${ }^{2}$ Institute of Microbiology and Infection, School of Biosciences, University of Birmingham, Edgbaston; Birmingham, United Kingdom.

${ }^{3}$ Center for Cell Dynamics and Department of Cell Biology, Johns Hopkins University School of Medicine; Baltimore, MD, USA.

${ }^{4}$ Department of Biomedical Engineering, Rutgers University; Piscataway, NJ, USA

${ }^{5}$ Division of Rheumatology, Department of Medicine, Johns Hopkins University School of Medicine; Baltimore, MD, USA.

${ }^{6}$ ImmunoTechnology Section, Vaccine Research Center, NIAID, NIH; Bethesda, MD, USA.

${ }^{7}$ Division of Transfusion Medicine, Department of Pathology, Johns Hopkins University School of Medicine; Baltimore, MD, USA.

${ }^{8}$ Department of Pediatrics, NYU Langone Medical Center, New York, NY and Maimonides Medical Center; Brooklyn, NY, USA.

${ }_{9}^{9}$ Department of Dermatology, George Washington University School of Medicine and Health Sciences; Washington, DC, USA.

${ }^{10}$ Division of Transfusion Medicine, Department of Pathology, Johns Hopkins University; Baltimore, MD, USA.

${ }^{11}$ Division of Infectious Diseases, Department of Medicine, Johns Hopkins University; Baltimore, MD, USA.

${ }^{12}$ Departments of Neurology and Neuroscience, Johns Hopkins University School of Medicine; Baltimore, MD, USA.

${ }^{13}$ Muscle Disease Unit, National Institute of Arthritis and Musculoskeletal and Skin Diseases, $\mathrm{NIH}$; Bethesda, MD, USA and Departments of Neurology and Medicine, Johns Hopkins

University School of Medicine; Baltimore, MD, USA.

$\dagger$ Co-first author

*Corresponding author:

H. Benjamin Larman, Ph.D.

Miller Research Building, Room 607

Baltimore, MD 21205

Phone: 410-614-6525

Email: hlarman1@jhu.edu 
bioRxiv preprint doi: https://doi.org/10.1101/2021.03.02.432977; this version posted March 3, 2021. The copyright holder for this preprint (which was not certified by peer review) is the author/funder, who has granted bioRxiv a license to display the preprint in perpetuity. It is made available under aCC-BY-ND 4.0 International license.

\section{Graphical Abstract}

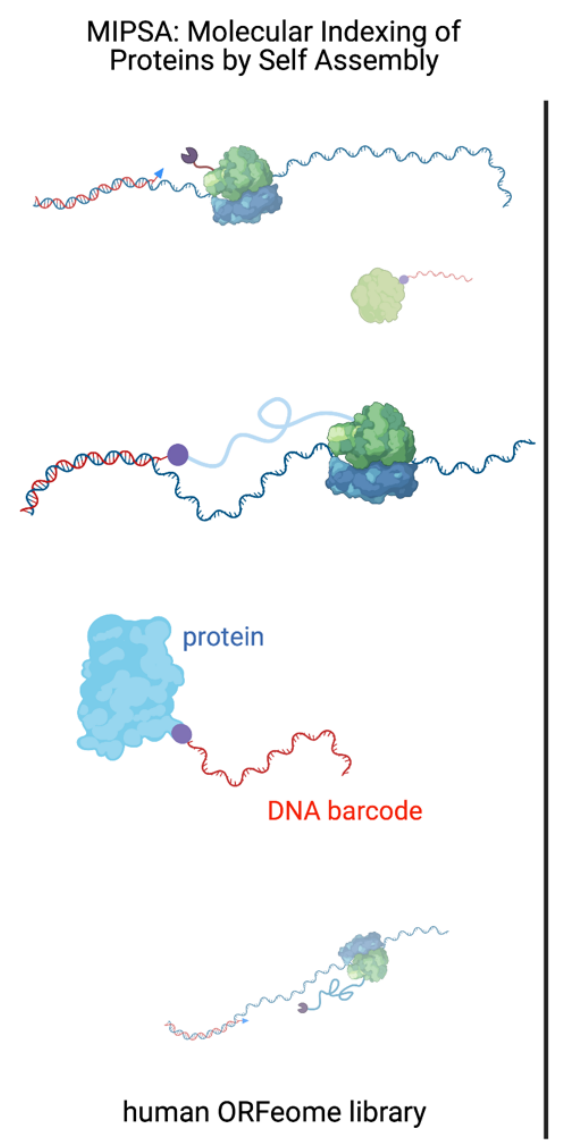

Immunoprecipitation with
plasma autoantibodies
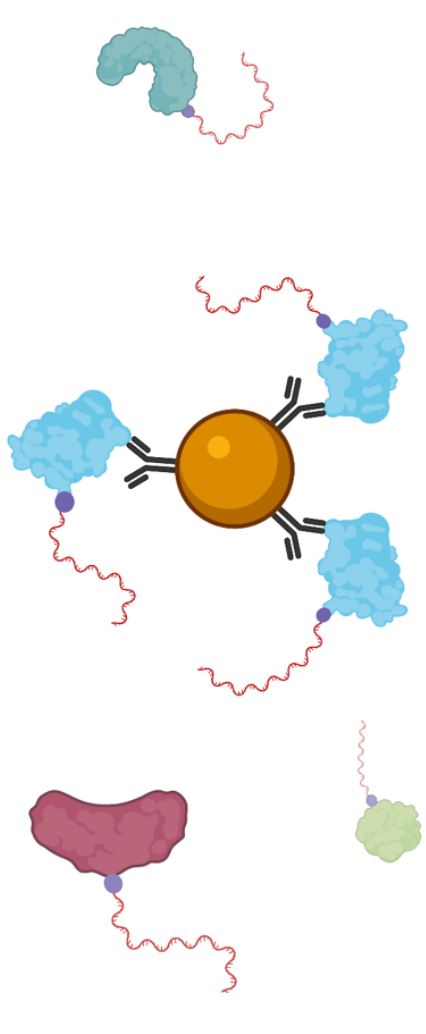

Neutralizing IFN- $\lambda 3$ antibodies

in severe COVID-19

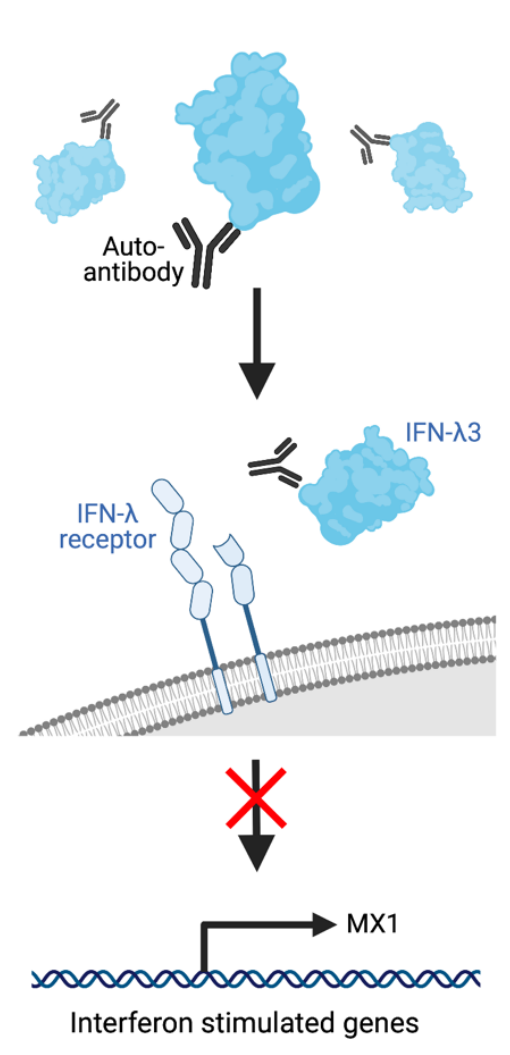




\begin{abstract}
Unbiased antibody profiling can identify the targets of an immune reaction. A number of likely pathogenic autoreactive antibodies have been associated with life-threatening SARS-CoV2 infection; yet, many additional autoantibodies likely remain unknown. Here we present

5 Molecular Indexing of Proteins by Self Assembly (MIPSA), a technique that produces ORFeomescale libraries of proteins covalently coupled to uniquely identifying DNA barcodes for analysis by sequencing. We used MIPSA to profile circulating autoantibodies from 55 patients with severe COVID-19 against 11,076 DNA-barcoded proteins of the human ORFeome library. MIPSA identified previously known autoreactivities, and also detected undescribed neutralizing interferon lambda 3 (IFN- $\lambda 3$ ) autoantibodies. At-risk individuals with anti- IFN- $\lambda 3$ antibodies may benefit from interferon supplementation therapies, such as those currently undergoing clinical evaluation.
\end{abstract}

\title{
One-Sentence Summary
}

Molecular Indexing of Proteins by Self Assembly (MIPSA) identifies neutralizing IFNL3

15 autoantibodies in patients with severe COVID-19. 


\section{Introduction}

Unbiased analysis of antibody binding specificities can provide important insights into health and disease states. We and others have utilized programmable phage display libraries to identify novel autoantibodies, characterize anti-viral immunity and profile allergen-specific IgE

5 antibodies.(1-4) While phage display has been useful for these and many other applications, most protein-protein, protein-antibody and protein-small molecule interactions require a degree of conformational structure that is not captured by displayed peptides. Profiling conformational protein interactions at proteome scale has traditionally relied on protein microarray technologies. Protein microarrays, however, tend to suffer from high per-assay cost, and a myriad of technical

10 artifacts, including those associated with the high throughput expression and purification of proteins, the spotting of proteins onto a solid support, the drying and rehydration of arrayed proteins, and the slide-scanning fluorescence imaging-based readout.(5, 6) Alternative approaches to protein microarray production and storage have been developed (e.g. Nucleic AcidProgrammable Protein Array, NAPPA(7) or single-molecule PCR-linked in vitro expression,

15 SIMPLEX (8)), but a robust, scalable, and cost-effective technology has been lacking.

To overcome the limitations associated with array-based profiling of full-length proteins, we previously established a methodology called ParalleL Analysis of Translated Open reading frames (PLATO), which utilizes ribosome display of open reading frame (ORF) libraries.(9) Ribosome display relies on in vitro translation of mRNAs that lack stop codons, stalling ribosomes suffers from several key limitations that have hindered its adoption. An ideal alternative is the covalent conjugation of proteins to short, amplifiable DNA barcodes. Indeed, individually prepared DNA-barcoded antibodies and proteins have been employed successfully in a myriad of applications, as reviewed recently by Liszczak and Muir.(10) One particularly attractive protein- 
DNA conjugation method involves the HaloTag system, which adapts a bacterial enzyme that forms an irreversible covalent bond with halogen-terminated alkane moieties.(11) Individual DNA-barcoded HaloTag fusion proteins have been shown to greatly enhance sensitivity and dynamic range of autoantibody detection, compared with traditional ELISA.(12) Scaling

5 individual protein barcoding to entire ORFeome libraries would be immensely valuable, but formidable due to high cost and low throughput. Therefore, a self-assembly approach could provide a much more efficient path to library production.

Here we describe a novel molecular display technology, Molecular Indexing of Proteins by Self Assembly (MIPSA), which overcomes key disadvantages of PLATO and other full-length protein array technologies. MIPSA produces libraries of soluble full-length proteins, each uniquely identifiable via covalent conjugation to a DNA barcode, flanked by universal PCR primer binding sequences. Barcodes are introduced near the 5' end of transcribed mRNA sequences, upstream of the ribosome binding site (RBS). Reverse transcription (RT) of the 5' end of in vitro transcribed RNA (IVT-RNA) creates a cDNA barcode, which is linked to a haloalkane-labeled RT primer. An

15 N-terminal HaloTag fusion protein is encoded downstream of the RBS, such that in vitro translation results in the intra-complex ("cis"), covalent coupling of the cDNA barcode to the HaloTag and its downstream open reading frame (ORF) encoded protein product. The resulting library of uniquely indexed full-length proteins can be used for inexpensive proteome-wide interaction studies, such as unbiased autoantibody profiling. We demonstrate the utility of the platform by uncovering known and novel autoantibodies in the plasma of patients with severe COVID-19. 


\section{Results}

Development of the MIPSA system

The MIPSA Gateway Destination vector contains the following key elements: a T7 RNA polymerase transcriptional start site, an isothermal unique clonal identifier ("UCI") barcode

5 sequence flanked by constant primer binding sites, a ribosome binding site (RBS), an N-terminal HaloTag fusion protein (891 nt), recombination sequences for ORF insertion, a stop codon, and a homing endonuclease site for plasmid linearization. A recombined ORF-containing pDESTMIPSA plasmid is shown in Fig. 1A.

We first sought to establish a library of pDEST-MIPSA plasmids containing stochastic, isothermal UCIs located between the transcriptional start site and the ribosome binding site. A degenerate oligonucleotide pool was synthesized, comprising melting temperature (Tm) balanced sequences: (SW)18-AGGGA-(SW) 18 , where $\mathrm{S}$ represents an equal mix of $\mathrm{C}$ and $\mathrm{G}$, while W represents an equal mix of $\mathrm{A}$ and $\mathrm{T}$ (Fig. 1B). We reasoned that this inexpensive pool of sequences would (i) provide sufficient complexity $\left(2^{36} \sim 7 \times 10^{10}\right)$ for unique ORF labeling, (ii) amplify without distortion, and (iii) serve as ORF-specific forward and reverse qPCR primer binding sites for measurement of individual UCIs of interest. The degenerate oligonucleotide pool was amplified by PCR, restriction cloned into the MIPSA destination vector, and transformed into $E$. coli (Methods). About 800,000 transformants were scraped off selection plates to obtain the pDEST-MIPSA UCI plasmid library. ORFs encoding the housekeeping gene glyceraldehyde-3phosphate dehydrogenase (GAPDH) and a known autoantigen, tripartite motif containing-21 (TRIM21, commonly known as Ro52), were separately recombined into the pDEST-MIPSA UCI plasmid library and used in the following experiments. Individually barcoded GAPDH and TRIM21 clones were isolated and sequenced. 


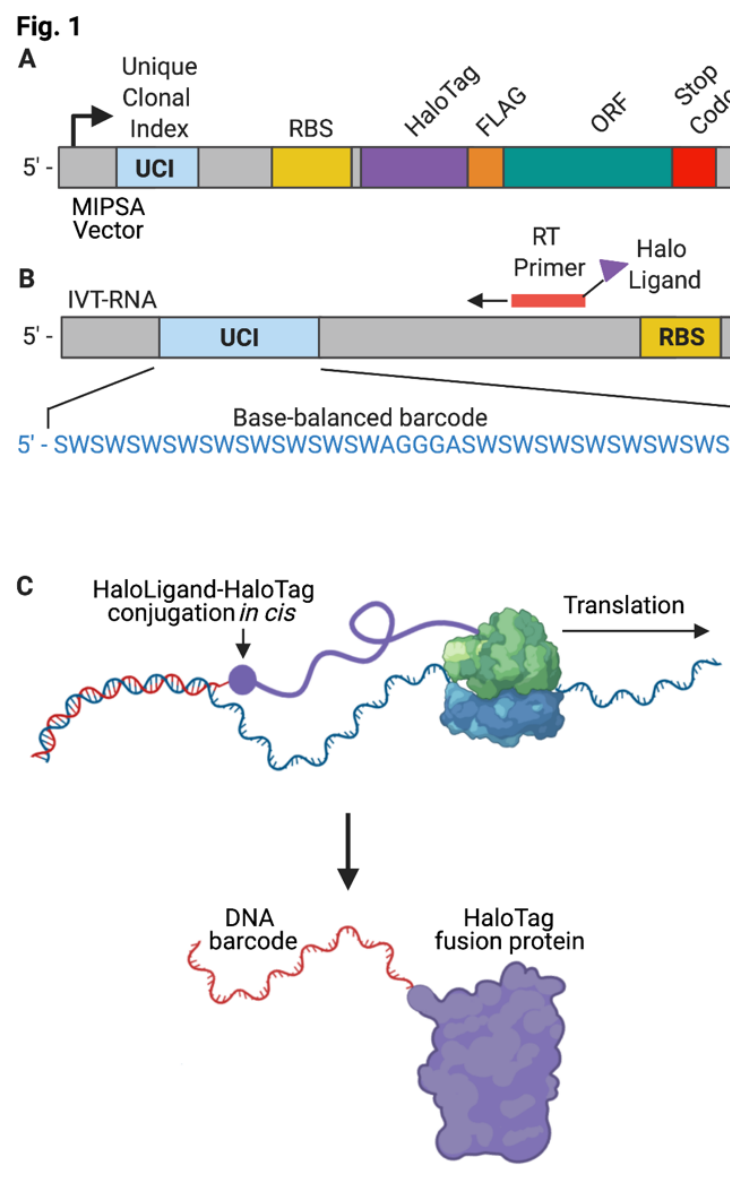

D

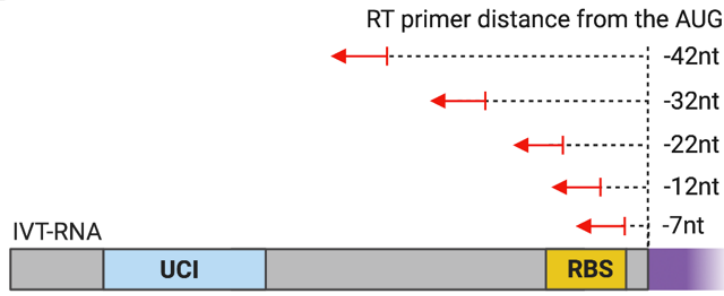

E
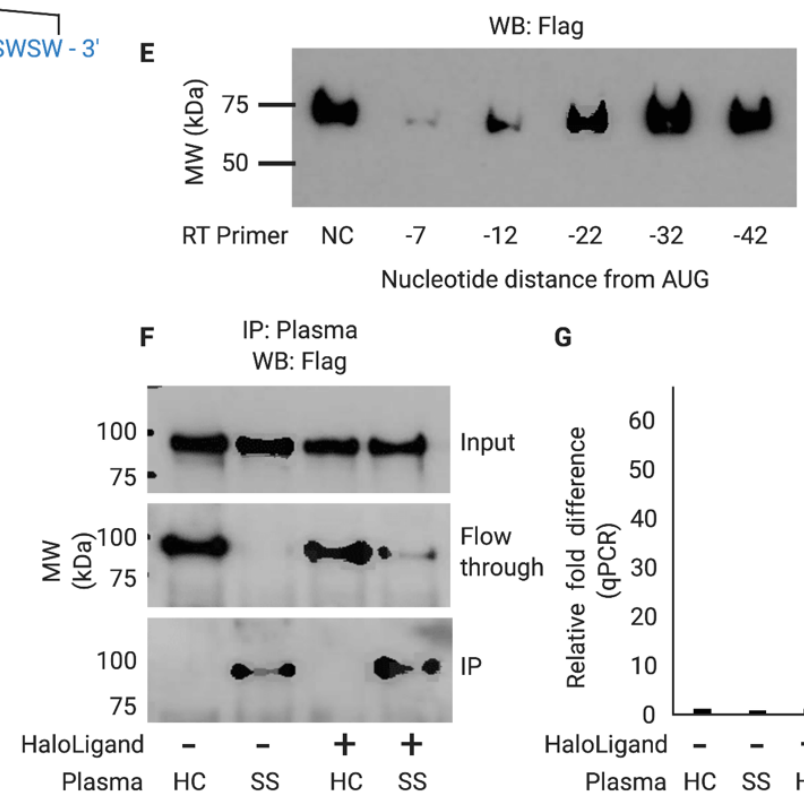

G

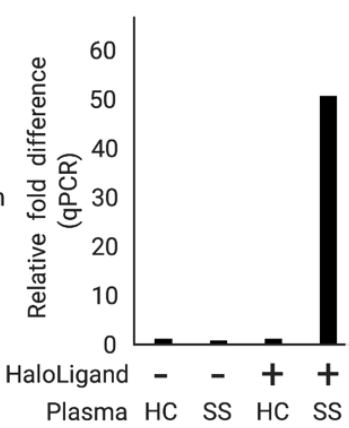

Fig. 1. The MIPSA method. (A) Schematic of the recombined pDEST-MIPSA vector with key components highlighted: unique clonal index (UCI, blue), ribosome binding site (RBS, yellow), N-terminal HaloTag (purple), FLAG epitope (orange), open reading frame (ORF, green), stop codon (red) and the I-SceI restriction endonuclease site (black) for vector linearization. (B) Schematic showing in vitro transcribed (IVT) RNA from the vector template shown in $\mathbf{A}$. Isothermal base-balanced UCI sequence: $(\mathrm{SW})_{18}$-AGGGA-(SW) 18 . (C) Cell-free translation of the RNA-cDNA shown in B. HaloTag protein forms a covalent bond with the HaloLigand-conjugated UCI-containing cDNA in cis during translation. (D) RT primer positions tested for impact on translation. (E) $\alpha$-FLAG western blot analysis of translation in presence of RT primers depicted in D (NC, negative control, no RT primer). (F) Western blot analysis of TRIM21 protein translated from RNA carrying the UCI-cDNA primed from the -32 position, either conjugated (+) or not (-) with the HaloLigand. Sjogren's Syndrome, SS; Healthy Control, HC. (G) qPCR analysis of the IPed TRIM21 UCI. Fold-difference is by comparison with the HaloLigand (-) HC IP. 
The MIPSA procedure involves RT of the stochastic barcode using a succinimidyl ester (O2)-haloalkane (HaloLigand)-conjugated RT primer. The bound RT primer should not interfere with the assembly of the E. coli ribosome and initiation of translation, but should be sufficiently proximal such that coupling of the HaloLigand-HaloTag-protein complex might hinder additional

5 rounds of translation. We tested a series of RT primers that anneal at distances ranging from -42 nucleotides to -7 nucleotides ( $5^{\prime}$ to $3^{\prime}$ ) relative to the zero position of the AUG start codon (Fig. 1D). Based on the yield of protein product from mRNA saturated with primers at these varying locations, we selected the -32 position as it did not interfere with translation efficiency (Fig. 1E). In contrast, RT from primers located within 20 nucleotides of the RBS diminished or abolished protein translation. This result agrees with the estimated footprint of assembled $70 \mathrm{~S} E$. coli ribosomes, which have been shown to protect a minimum of 15 nucleotides of mRNA.(13)

We next assessed the ability of SuperScript IV to perform RT from a primer labeled with the HaloLigand at its 5' end, and the ability of the HaloTag-TRIM21 protein to form a covalent bond with the HaloLigand-conjugated primer during the translation reaction. HaloLigand 15 conjugation and purification followed Gu et al. (Methods, Fig. S1).(14) Either an unconjugated RT primer or a HaloLigand-conjugated RT primer was used for RT of the barcoded HaloTagTRIM21 mRNA. The translation product was then immunoprecipitated (IPed) with plasma from a healthy donor or plasma from a TRIM21 (Ro52) autoantibody-positive patient with Sjogren's Syndrome (SS). The SS plasma efficiently IPed the TRIM21 protein, regardless of RT primer conjugation, but only pulled down the TRIM21 cDNA UCI when the HaloLigand-conjugated primer was used in the RT reaction (Fig. 1F-G). 


\section{Assessing cis versus trans UCI barcoding}

While the previous experiment indicated that indeed the HaloLigand does not impede RT priming, and that the HaloTag can form a covalent bond with the HaloLigand during the translation reaction, it did not elucidate the amount of cis (intra-complex) versus trans (inter-complex)

5 HaloTag-UCI conjugation (Fig. S2). Here, "intra-complex" is defined as conjugation to the UCI, which is bound to the same RNA encoding the protein. To measure the amount of cis and trans HaloTag-UCI conjugation, GAPDH and TRIM21 mRNAs were separately reverse transcribed (using HaloLigand-conjugated primer) and then either mixed 1:1 or kept separate for in vitro translation. As expected, translation of the mixture produced roughly equivalent amounts of each protein compared to the individual translations (Fig. S3). SS plasma specifically IPed TRIM21 protein regardless of translation condition (Fig. S3, IPed fraction). However, we noted that while the SS IPs contained high levels of the TRIM21 UCI, as intended, more of the GAPDH UCI was pulled down by the SS plasma compared to that by the HC plasma when the mRNA was mixed prior to translation. This indicates that indeed some trans barcoding occurs (Fig. 2A). We estimate that $\sim 50 \%$ of the protein is cis-barcoded, with the remaining $50 \%$ trans-barcoded protein equally represented by both UCIs. Thus, in this two-component system, $25 \%$ of the TRIM21 protein is conjugated to the GAPDH UCI.

In the setting of a complex library, even if $\sim 50 \%$ of the protein is trans barcoded, this side product is uniformly distributed across all members of the library. We tested this hypothesis using a model MIPSA library composed of 100-fold excess of a second GAPDH clone, which was combined with a 1:1 mixture of the first GAPDH and TRIM21 clones (Fig. 2B). We additionally developed a sequencing workflow utilizing a PCR spike-in sequence for absolute quantification of each UCI. IP with SS plasma resulted in the specific IP of the TRIM21-UCI, with negligible transcoupled GAPDH-UCI detected (Fig. 2B). Using the spiked-in sequence for absolute 
quantification, and assuming of $100 \%$ of the input TRIM21 protein in the IP fraction, we calculated a cis coupling efficiency of about $0.2 \%$ (i.e. $0.2 \%$ of input TRIM21 RNA molecules were converted into the intended cis UCI-coupled TRIM21 proteins).
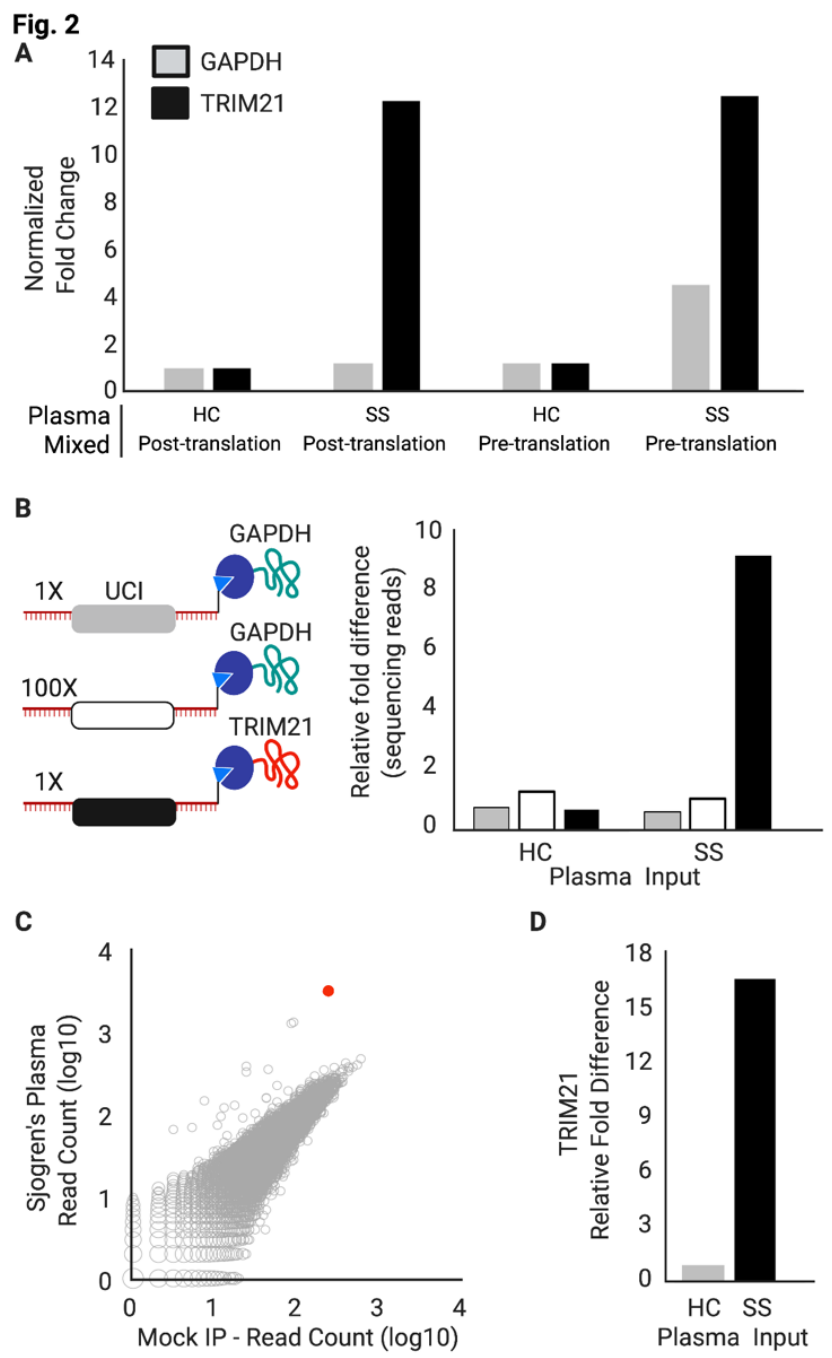

5 Fig. 2. Cis- versus trans-UCI conjugation. (A) IVT-RNA encoding TRIM21 or GAPDH with their distinct UCI barcodes were translated before or after mixing at a 1:1 ratio. qPCR analysis of the IPs using UCI-specific primers, reported as fold-change versus IP with HC plasma, when the IVT-RNA was mixed post-translation. (B) IVT-RNA encoding TRIM21 (black UCI) and GAPDH (gray UCI) were mixed 1:1 into a background of 100-fold excess GAPDH (white UCI) and then translated. Sequencing analysis of the IPs, reported as fold-change versus the HC IP of the 100x GAPDH. (C) hORFeome MIPSA library containing spiked-in TRIM21, IPed with SS plasma and compared to average of 8 mock IPs (no plasma input). The TRIM21 UCI is shown in red. (D) Relative fold difference of TRIM21 UCI in SS vs HC IPs, determined by sequencing. 


\section{Establishing and deconvoluting a stochastically barcoded human ORFeome MIPSA library}

The sequence-verified human ORFeome (hORFeome) v8.1 is composed of 12,680 clonal ORFs mapping to 11,437 genes in pDONR223.(15) Five subpools of the library were created, each composed of $\sim 2,500$ similarly sized ORFs. Each of the five subpools was separately recombined

$5 \quad$ into the pDEST-MIPSA UCI plasmid library and transformed to obtain $\sim 10$-fold ORF coverage ( 25,000 clones per subpool). Each subpool was assessed via Bioanalyzer electrophoresis, sequencing of $\sim 20$ colonies, and Illumina sequencing of the superpool. The TRIM21 plasmid was spiked into the superpooled hORFeome library at 1:10,000 - comparable to a typical library member. The SS IP experiment was then performed on the hORFeome MIPSA library, using sequencing as a readout. The reads from all barcodes in the library, including the spiked-in TRIM21, are shown in Fig. 2C. The SS autoantibody-dependent enrichment of TRIM21 (17-fold) was similar to the model system (Fig. 2D). Assuming the coupling efficiencies derived earlier, we estimate that about $6 \times 10^{5}$ correctly cis-coupled TRIM21 molecules (and thus each library member on average) was input to the IP reaction.

Next, we established a system for creating a UCI-ORF lookup dictionary, using tagmentation and sequencing (Fig. 3A). Sequencing the 5' $50 \mathrm{nt}$ of the ORF inserts detected 11,076 of the 11,887 unique 5' $50 \mathrm{nt}$ sequences. Of the 153,161 unique barcodes detected, $82.9 \%$ $(126,975)$ were found to be associated with a single ORF ("monoclonal”). Each ORF was uniquely associated with a median of 9 (ranging from 0 to 123) UCIs (Fig. 3B). Aggregating the reads corresponding to each ORF, over $99 \%$ of the represented ORFs were present within a 10 -fold difference of the median ORF abundance (Fig. 3C). Taken together, these data indicated that we established a uniform library of 11,076 stochastically indexed human ORFs, and sufficiently defined a lookup dictionary for downstream analyses. Fig. 3D shows a SS IP versus mock IP, but 
with the 47 dictionary-decoded GAPDH UCIs (corresponding to two GAPDH isoforms present in the hORFeome library) appearing along the $\mathrm{y}=\mathrm{x}$ diagonal as expected.

Fig. 3

MIPSA Library
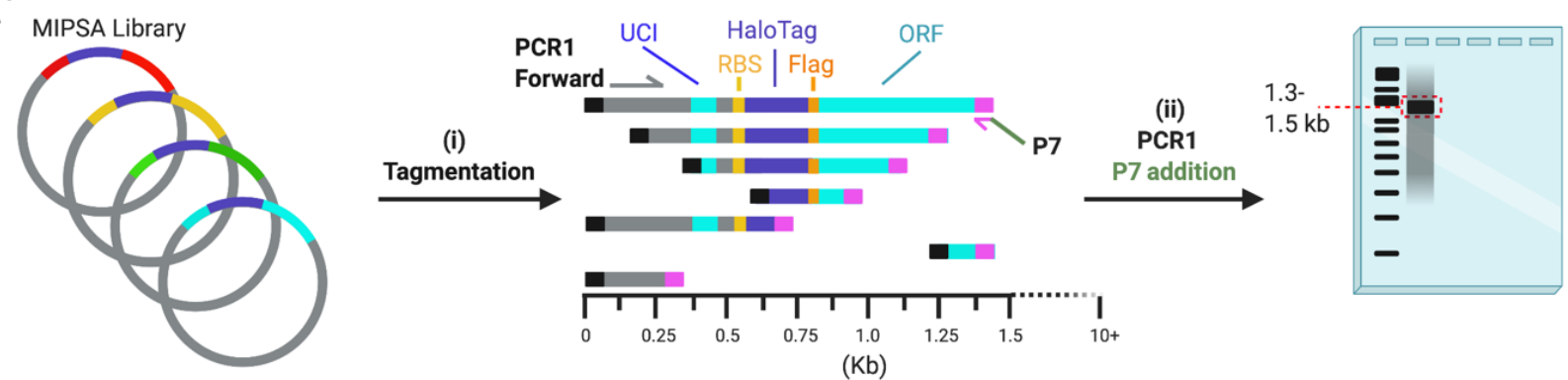

(Kb)
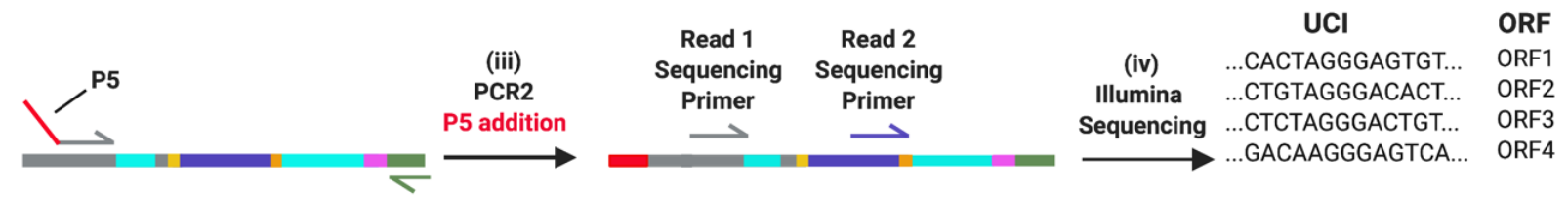

B

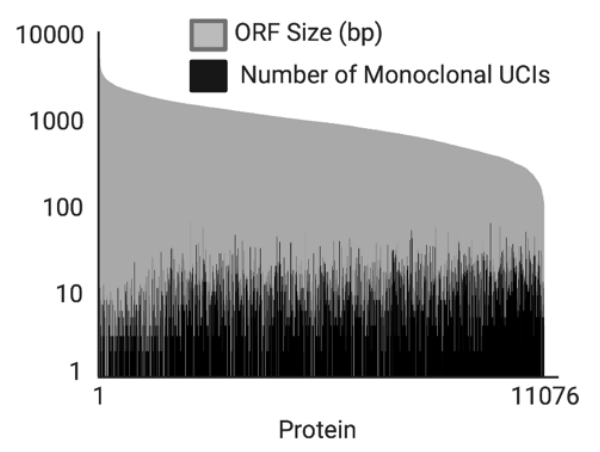

C

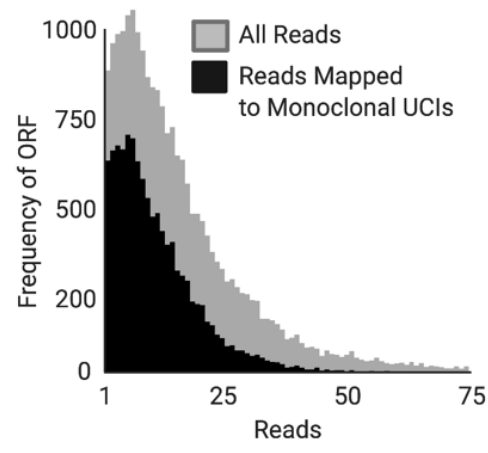

D

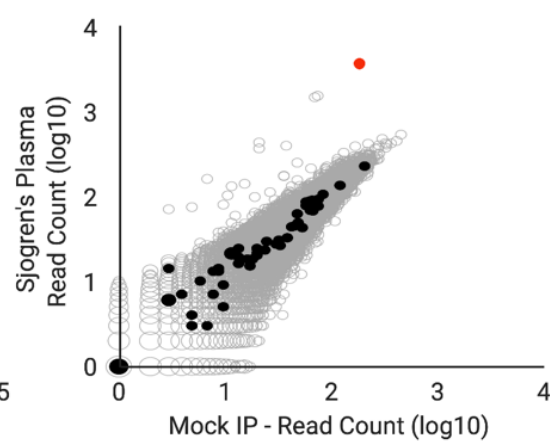

Fig. 3. Defining the UCI-ORF dictionary. (A) Tagmentation randomly inserts adapters into the MIPSA vector library. Utilizing a PCR1 forward primer and the reverse primer of the tagmentation-inserted adapter, DNA fragments are amplified and size selected to be $\sim 1.5 \mathrm{~kb}$, which captures the 5' terminus of the ORF. These fragments are amplified with a P5-containing PCR2 forward primer and a P7 reverse primer. Illumina sequencing is used to read the UCI and the ORF from the same fragment, thus enabling their association in the dictionary. (B) The number of uniquely-associated UCIs is shown for each member of pDEST-MIPSA hORFeome library, superimposed on the length of the ORF. (C) Distribution of reads associated with each ORF, both total reads and UCI-matched reads. (D) IP of hORFeome MIPSA library using Sjogren's Syndrome (SS) plasma is compared to the average of 8 mock IPs. Sequencing reads of each UCI are plotted. UCIs associated with the two library GAPDH isoforms (filled black) and spiked-in TRIM21 (red) are indicated. 


\section{Unbiased MIPSA analysis of autoantibodies associated with severe COVID-19}

Several recent reports have described elevated autoantibody reactivities in patients with severe COVID-19.(16-20) We therefore used MIPSA with the human ORFeome library for unbiased identification of autoreactivities in the plasma of 55 severe COVID-19 patients. For

5 comparison, we used MIPSA to detect autoreactivities in plasma from 10 healthy donors and 10 COVID-19 convalescent plasma donors who had not been hospitalized (Table S1). Each sample was compared to a set of 8 "mock IPs", which contained all reaction components except for plasma. Comparison to mock IPs accounts for bias in the library and background binding. Importantly, the informatic pipeline used to detect antibody-dependent reactivity (Methods) yielded a median of 5 (ranging from 2 to 9) false positive UCI hits per mock IP. IPs using plasma from severe COVID-19 patients, however, yielded a mean of 132 reactive UCIs, significantly more than the mean of 93 reactive UCIs among the controls $(\mathrm{p}=0.018$, $\mathrm{t}$-test $)$. Collapsing UCIs to their corresponding proteins yielded a mean of 83 reactive proteins among severe COVID-19 patients, which was significantly more than the mean of 63 reactive proteins among controls $($ Fig. $\mathbf{4 A}, \mathrm{p}=$ $0.019, \mathrm{t}$-test).

We next examined proteins in the severe COVID-19 IPs that had at least two reactive UCIs (in the same IP), which were reactive in at least one severe patient, and that were not reactive in more than one control (healthy or mild/moderate convalescent plasma). Proteins were excluded if they were reactive in a single severe patient and a single control. The 103 proteins that met these patients exhibited reactivity to at least one of these proteins. We noted co-occurring protein reactivities in multiple individuals, the vast majority of which lack homology by protein sequence alignment. Table $\mathbf{S 2}$ provides summary statistics about these reactive proteins, including whether 
they are previously defined autoantigens according to the human autoantigen database AAgAtlas

1.0.(21) Data S1 provides the patient versus UCI-level data used to construct the heatmap.

Fig. 4

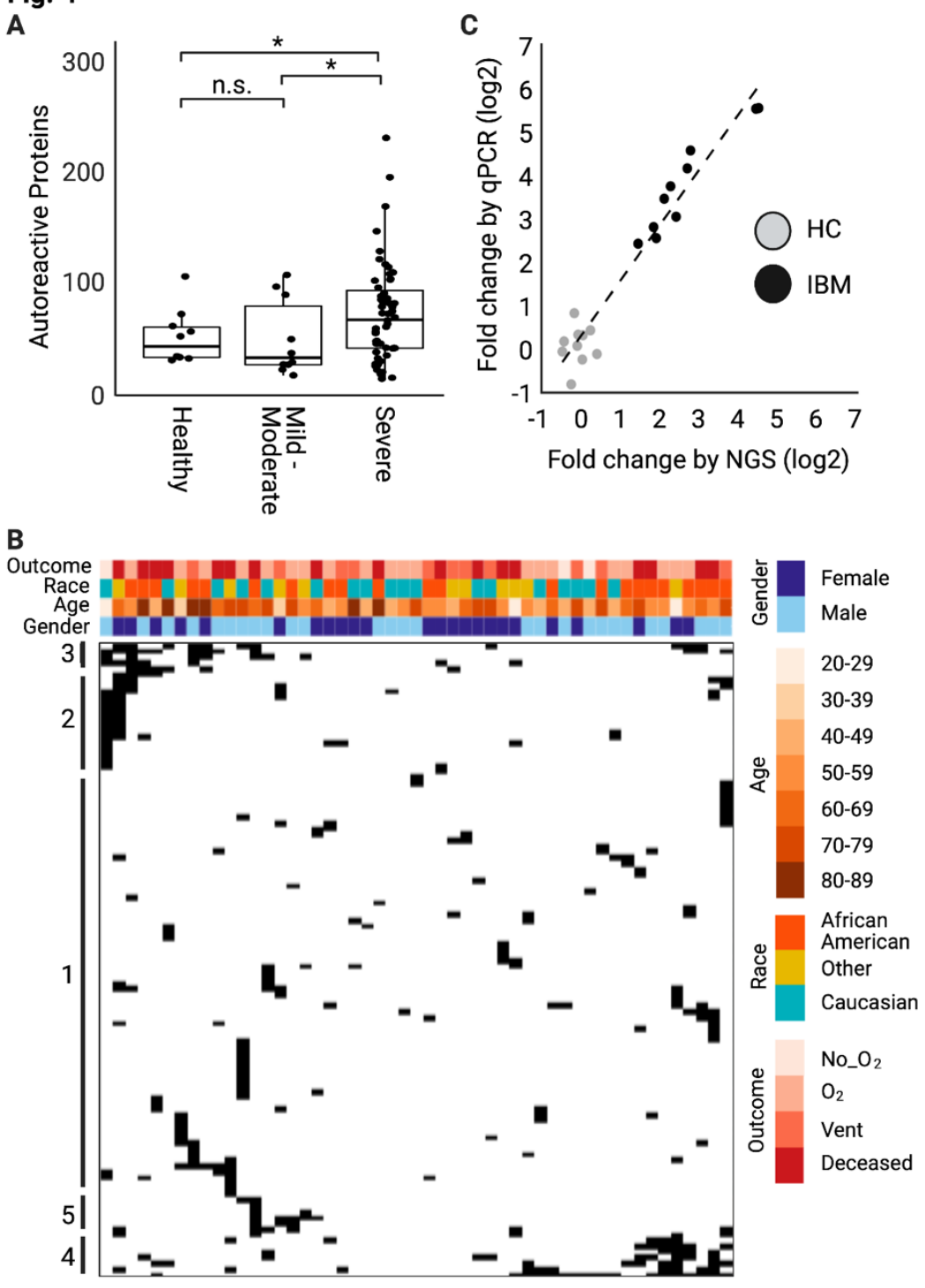

Fig. 4. MIPSA analysis of autoantibodies in severe COVID-19. (A) Boxplots showing total numbers of reactive proteins in plasma from healthy controls, mild-moderate COVID-19 patients, or severe COVID-19 patients. * indicates $\mathrm{p}<0.05$ using t-test to compare means. (B) Hierarchal clustering of all proteins represented by at least 2 reactive UCIs in at least 1 severe COVID-19 plasma, but not more than 1 control (healthy or mild-moderate COVID-19 plasma). (C) MIPSA analysis of autoantibodies in 10 Inclusion Body Myositis (IBM) patients and 10 healthy controls (HCs), using the hORFeome library. Fold change of IPed 5'-nucleotidase, cytosolic 1A (NT5C1A), measured by UCI-qPCR (relative to average of $10 \mathrm{HCs}$ ) or sequencing (relative to mock IPs). 
One notable autoreactivity cluster (Table S2, cluster \#5) includes 5'-nucleotidase, cytosolic 1A (NT5C1A), which is highly expressed in skeletal muscle and is the most well-characterized autoantibody target in inclusion body myositis (IBM). Multiple UCIs linked to NT5C1A were significantly increased in 3 of the 55 severe COVID-19 patients (5.5\%). NT5C1A autoantibodies

5 have been reported in up to $70 \%$ of IBM patients (1), in $20 \%$ of Sjogren's Syndrome (SS) patients, and in up to $\sim 5 \%$ of healthy donors.(22) The prevalence of $\mathrm{NT} 5 \mathrm{C} 1 \mathrm{~A}$ reactivity in the severe COVID-19 cohort is therefore not necessarily elevated. However, we wondered whether MIPSA would be able to reliably distinguish between healthy donor and IBM plasma based on NT5C1A reactivity. We tested plasma from 10 healthy donors and 10 IBM patients, the latter of whom were selected based on NT5C1A seropositivity determined by PhIP-Seq.(1) The clear separation of patients from controls in this independent cohort suggests that MIPSA may indeed have utility in clinical diagnostic testing using either UCI-specific qPCR or library sequencing, which were tightly correlated readouts (Fig. 4C).

Neutralizing autoantibodies targeting type I interferons alpha (IFN- $\alpha$ ) and omega (IFN- $\omega)$ have been associated with severe COVID-19.(17, 23, 24) All type I interferons except IFN- $\alpha 16$ are represented in the human MIPSA library and dictionary. However, IFN- $\alpha 4$, IFN- $\alpha 17$, and IFNa21 are indistinguishable by the first 50 nucleotides of their encoding ORF sequences. Two of the severe COVID-19 patients in this cohort (3.6\%) exhibited dramatic IFN- $\alpha$ autoreactivity (43 and 41 IFN- $\alpha$ UCIs, across 10 distinct ORFs, along with 5 and 2 IFN- $\omega$ UCIs, Fig. 5A-B). The extensive co-reactivity of these proteins is likely attributable to their sequence homology (Fig. S4). By requiring at least 2 IFN UCIs to be considered positive, we identified two additional severe COVID-19 plasma (P3-P4) with lower levels of IFN- $\alpha$ reactivity, each with only 2 reactive IFN- 
bioRxiv preprint doi: https://doi.org/10.1101/2021.03.02 432977; this version posted March 3, 2021. The copyright holder for this preprint (which was not certified by peer review) is the author/funder, who has granted bioRxiv a license to display the preprint in perpetuity. It is made available under aCC-BY-ND 4.0 International license.

$\alpha$ UCIs. Interestingly, one additional plasma (P5) precipitated five UCIs from the type III interferon IFN- $\lambda 3$, but no UCI from any type I or II interferon (Fig. 5C-D). None of the healthy or non-hospitalized COVID-19 controls were positive for 2 or more interferon UCIs.
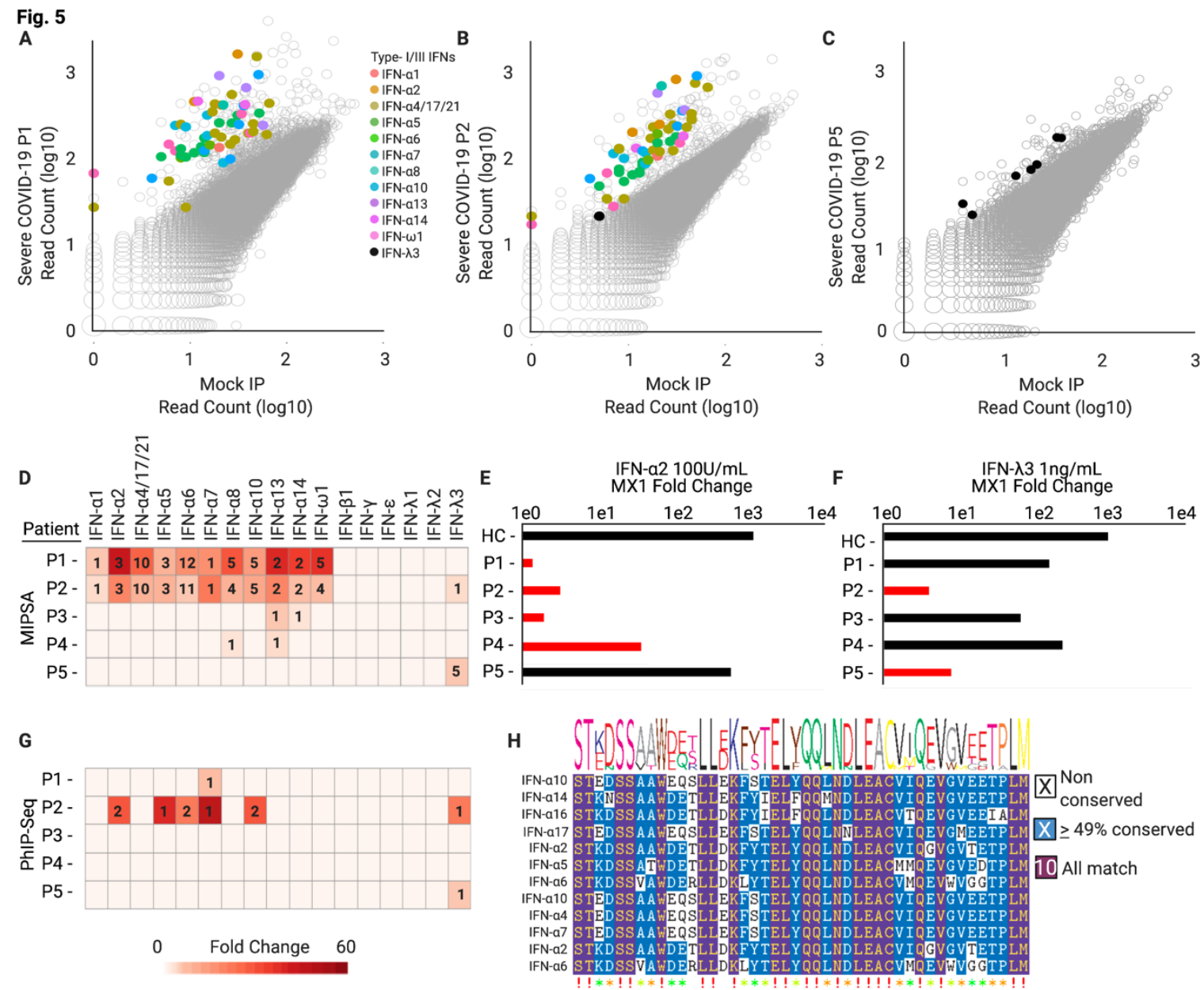

5 Fig. 5. MIPSA detects known and novel neutralizing interferon autoantibodies. (A-C) Scatterplots highlighting reactive interferon UCIs for three severe COVID-19 patients. (D) Summary of interferon reactivity detected in 5 of 55 individuals with severe COVID-19. Hits foldchange values (cell color) and the number of reactive UCIs (number in cell) are provided. (E, F) Recombinant interferon alpha 2 (IFN- $\alpha 2$ ) or interferon lambda 3 (IFN- $\lambda 3$ ) neutralizing activity of the same patients shown in D. Plasma were pre-incubated with $100 \mathrm{U} / \mathrm{ml}$ of IFN- $\alpha 2$ or $1 \mathrm{ng} / \mathrm{ml}$ of IFN- $\lambda 3$ prior to incubation with A549 cells. Fold changes of the interferon stimulated gene, MX1, were calculated by RT-qPCR relative to unstimulated cells. GAPDH was used as a housekeeping control gene for normalization. Red bars indicate which samples are predicted by MIPSA to have neutralizing activity for each interferon. (G) PhIP-Seq analysis of interferon autoantibodies in the 5 patients of $\mathbf{D}$ (row and column orders maintained). Hits fold-change values (cell color) and the number of reactive peptides (number in cell) are provided. (H) Epitopefindr analysis of the PhIPSeq reactive type I interferon 90 -aa peptides. 
Incubation of A549 human adenocarcinomatous lung epithelial cells with $100 \mathrm{U} / \mathrm{ml}$ IFN- $\alpha$ or $1 \mathrm{ng} / \mathrm{ml}$ of IFN- $\lambda 3$ for 4 hours in serum-free medium resulted in a robust upregulation of the IFN-response gene MX1 by $\sim 1,000$-fold and 100-fold, respectively. Pre-incubation of the IFN-

$5 \quad \alpha 2$ with plasma P1, P2, or P3 completely abolished the A549 interferon response (Fig. 5E). The plasma with the weakest IFN- $\alpha$ reactivity by MIPSA (P4) partially neutralized the cytokine. Neither HC nor P5 plasma had any effect on the response of A549 cells to IFN- $\alpha 2$. However, preincubation of the IFN- $\lambda 3$ with the MIPSA-reactive plasma, P2 and P5, neutralized the cytokine (Fig. 5F). None of the other plasma (HC, P1, P3, or P4) had any effect on the response of A549 10 cells to IFN- $\lambda 3$. In summary, antibody profiling of this severe COVID-19 cohort identified strongly neutralizing IFN- $\alpha$ autoantibodies in $5.5 \%$ of patients and strongly neutralizing IFN- $\lambda 3$ autoantibodies in $3.6 \%$ of patients, with a single patient $(1.8 \%)$ harboring both autoreactivities.

We then asked if PhIP-Seq with a 90-aa human peptidome library(25) might also detect interferon antibodies in this cohort. PhIP-Seq detected IFN- $\alpha$ reactivity in plasma from P1 and P2, although to a much lesser extent (Fig. 5G). The two weaker IFN- $\alpha$ reactivities detected by MIPSA in the plasma of P3 and P4 were both missed by PhIP-Seq. PhIP-Seq identified a single additional weakly IFN- $\alpha$ reactive sample, which was negative by MIPSA (not shown). Detection of type III interferon autoreactivity (directed exclusively at IFN- $\lambda 3$ ) agreed perfectly between the two technologies. PhIP-Seq data was used to narrow the location of a dominant epitope in these type I and type III interferon autoantigens (Fig. 5H for IFN- $\alpha$, amino acid position 45-135 for IFN- $\lambda 3$ ).

We next wondered about the prevalence of the IFN- $\lambda 3$ autoreactivity in the general population, and whether it might be increased among patients with severe COVID-19. PhIP-Seq was used to profile the plasma of 423 healthy controls, none of whom were found to have detectable IFN- $\lambda 3$ autoreactivity. These data suggest that IFN- $\lambda 3$ autoreactivity may be more 
frequent among individuals with severe COVID-19. This is the first report describing neutralizing IFN- $\lambda 3$ autoantibodies, and therefore proposes a potentially novel pathogenic mechanism contributing to life-threatening COVID-19 in a subset of patients.

\section{Discussion}

Here we present a novel molecular display technology for full length proteins, which provides key advantages over protein microarrays, PLATO, and alternative techniques. MIPSA utilizes self-assembly to produce a library of proteins, linked to relatively short (158 nt) single stranded DNA barcodes via the $25 \mathrm{kDa}$ HaloTag domain. This compact barcoding approach will likely have numerous applications not accessible to alternative display formats with bulky linkage cargos (e.g. yeast, bacteria, viruses, phage, ribosomes, mRNAs). Indeed, individually conjugating minimal DNA barcodes to proteins, especially antibodies and antigens, has already proven useful in several contexts, including CITE-Seq,(26) LIBRA-seq,(27) and related methodologies.(23, 28) At proteome scale, MIPSA enables unbiased analyses of protein-antibody, protein-protein, and

15 protein-small molecule interactions, as well as studies of post-translational modification, such as hapten modification studies or protease activity profiling, for example. Key advantages of MIPSA include its high throughput, low cost, simple sequencing library preparation, and stability of the protein-DNA complexes (important for both manipulation and storage of display libraries). Importantly, MIPSA can be immediately adopted by standard molecular biology laboratories, DNA sequencing instrument or facility. 


\section{Complementarity of MIPSA and PhIP-Seq}

Display technologies frequently complement one another, but may not be amenable to routine use in concert. MIPSA is more likely than PhIP-Seq to detect antibodies directed at conformational epitopes on proteins expressed well in vitro. This was exemplified by the robust

5 detection of interferon alpha autoantibodies via MIPSA, which were less sensitively detected via PhIP-Seq. PhIP-Seq, on the other hand, is more likely to detect antibodies directed at less conformational epitopes contained within proteins that are either absent from an ORFeome library or cannot be expressed well in cell free lysate. Because MIPSA and PhIP-Seq naturally complement one another in these ways, we designed the MIPSA UCI amplification primers to be the same as those we have used for PhIP-Seq. Since the UCI-protein complex is stable - even in bacterial phage lysate - MIPSA and PhIP-Seq can readily be performed together in a single reaction, using a single set of amplification and sequencing primers. The compatibility of these two display modalities will therefore lower the barrier to leveraging their synergy.

A key aspect of MIPSA involves the conjugation of a protein to its associated UCI in cis, compared to another library member's UCI in trans. Here we have utilized covalent conjugation via the HaloTag/HaloLigand system, but there are others that could work as well. For instance, the SNAP-tag (a $20 \mathrm{kDa}$ mutant of the DNA repair protein O6-alkylguanine-DNA alkyltransferase) the RT primer in place of the HaloLigand. A mutant derivative of the SNAP-tag, the CLIP-tag, binds O2-benzylcytosine derivatives, which could also be adapted to MIPSA.(30)

The rate of fusion tag maturation and ligand binding is critical to the relative yield of cis versus trans UCI conjugation. A study by Samelson et al. determined that the rate of HaloTag 
protein production is about fourfold higher than the rate of HaloTag functional maturation.(31) Considering a typical protein size is $<1,000$ amino acids in the ORFeome library, these data predict that most proteins would be released from the ribosome before HaloTag maturation and thus before cis HaloLigand conjugation could occur, thereby favoring unwanted trans barcoding. However,

5 we observed $\sim 50 \%$ of protein-UCI conjugates are formed in cis, thereby enabling sufficient assay performance in the setting of a complex library. During optimization experiments, we found the rate of cis barcoding to be slightly improved by excluding release factors from the translation mix, which stalls ribosomes on their stop codons and allows HaloTag maturation to continue in proximity to its UCI. Alternative approaches to promote controlled ribosomal stalling could 10 include stop codon removal/suppression or use of a dominant negative release factor. Ribosome release could then be induced via addition of the chain terminator puromycin.

Since UCI cDNAs are formed on the 5' UTR of the IVT-RNA, eukaryotic ribosomes would be unable to scan from the 5' cap to the initiating Kozak sequence. The MIPSA system described here is therefore incompatible with cap-dependent cell-free translation systems. In case capdependent translation is required, however, two alternative methods could be developed. First, the current 5' UCI system could be used if an internal ribosome entry site (IRES) were to be placed between the RT primer and the Kozak sequence. Second, the UCI could instead be situated at the 3' end of the RNA, provided that the RT was prevented from extending into the ORF. Beyond cell-free translation, if either of these approaches were developed, RNA-cDNA hybrids could be transfected into living cells or tissues, where UCI-protein formation could take place in situ.

The ORF-associated UCIs can be embodied in a variety of ways. Here, we have stochastically assigned indexes to the human ORFeome at $\sim 10 \mathrm{x}$ representation. This approach has two main benefits, first being the low cost of the single degenerate oligonucleotide pool, and second being the multiple, independent pieces of evidence reported by the set of UCIs associated 
with each ORF. We have designed our library of stochastic barcodes to feature base-balanced sequences of uniform melting temperature, and thus more uniform PCR amplification efficiency. For simplicity, we have opted not to incorporate unique molecular identifiers (UMIs) into the primer, but this approach is compatible with MIPSA UCIs, and may potentially enhance

5 quantitation. One disadvantage of stochastic indexing is the potential for ORF dropout, and thus the need for relatively high UCI representation; this increases the depth of sequencing required to quantify each UCI, and thus the overall per-sample cost. A second disadvantage is the requirement to construct a UCI-ORFeome matching dictionary. With short-read sequencing, we were unable to disambiguate a fraction of the library, comprised mostly of alternative isoforms. Using a longread sequencing technology, such as PacBio or Oxford Nanopore Technologies, instead of or in addition to short-read sequencing technology could surmount incomplete disambiguation. As opposed to stochastic barcoding, individual UCI-ORF cloning is possible but costly and cumbersome. However, a smaller UCI set would provide the advantage of lower per-assay sequencing cost. We have previously developed a methodology to clone ORFeomes using Long

15 Adapter Single Stranded Oligonucleotide (LASSO) probes.(32) Incorporating target-specific indexes into the capture probe library would result in uniquely indexed ORFs, without dramatically increasing the cost of the LASSO probe library. LASSO cloning of ORFeome libraries may therefore synergize with MIPSA-based applications.

A useful feature of appropriately designed UCIs is that they can also serve as qPCR readout probes. The degenerate UCIs that we have designed and used here (Fig. 1B) also comprise $18 \mathrm{nt}$ base-balanced forward and reverse primer binding sites. The low cost and rapid turnaround time of a qPCR assay can thus be leveraged in combination with MIPSA. For example, incorporating 
assay quality control measures, such as the TRIM21 IP, can be used to qualify a set of samples prior to a more costly sequencing run. Troubleshooting and optimization can similarly be expedited by employing qPCR as a readout, rather than relying exclusively on NGS. qPCR testing of specific UCIs may theoretically also provide enhanced sensitivity compared to sequencing, and

$5 \quad$ may be more amenable to analysis in a clinical setting.

Autoantibodies detected in severe COVID-19 patients using MIPSA

The association between autoimmunity and severe COVID-19 disease is increasingly appreciated. In a cohort of 55 hospitalized individuals, we detected multiple established autoantibodies, including one that we have previously linked to inclusion body myositis.(1) We then tested the performance of MIPSA for detecting the NT5C1A autoantibody in a separate cohort of seropositive IBM patients and healthy controls. The results support future efforts in evaluating the clinical utility of MIPSA for standardized, comprehensive autoantibody testing. Such tests could utilize either single-plex qPCR or library sequencing as a readout. role, if any, they may play in severe COVID-19. In larger scale studies, we expect that patterns of co-occurring reactivity, or reactivities towards proteins with related biological functions, may ultimately define new autoimmune syndromes associated with severe COVID-19. Neutralizing IFN- $\alpha / \omega$ autoantibodies have been described in patients with severe COVID-19 disease and are presumed to be pathogenic.(17) These likely pre-existing autoantibodies, which occur very rarely in the general population, block restriction of viral replication in cell culture, and are thus likely to interfere with disease resolution. This discovery paved the way to identifying a subset of individuals at risk for life-threatening COVID-19, and proposed therapeutic use of interferon beta in this population of patients. In our study, MIPSA identified two individuals with extensive 
reactivity to the entire family of IFN- $\alpha$ cytokines. Indeed, plasma from both individuals, plus one individual with weaker IFN- $\alpha$ reactivity detected by MIPSA, robustly neutralized recombinant IFN- $\alpha 2$ in a lung adenocarcinomatous cell culture model. Unexpectedly, one individual in the cohort without IFN- $\alpha$ reactivity pulled down 5 IFN- $\lambda 3$ UCIs. A second, IFN- $\alpha$ autoreactive 5 individual, also pulled down a single IFN- $\lambda 3$ UCI. The same autoreactivities were also detected using PhIP-Seq. Interestingly, neither MIPSA nor PhIP-Seq detected reactivity to IFN- $\lambda 2$, despite their high degree of sequence homology (Fig. S4). We tested the IFN- $\lambda 3$ neutralizing capacity of these patients' plasma, observing near complete ablation of the cellular response to the recombinant cytokine (Fig. 5F). These data propose IFN- $\lambda 3$ autoreactivity is a new, potentially pathogenic mechanism contributing to severe COVID-19 disease.

Type III IFNs (IFN- $\lambda$, also known as IL-28/29) are cytokines with potent anti-viral activities that act primarily at barrier sites. The IFN- $\lambda$ R1/IL-10RB heterodimeric receptor for IFN$\lambda$ is expressed on lung epithelial cells and is important for the innate response to viral infection. Mordstein et al., determined that in mice, IFN- $\lambda$ diminished pathogenicity and suppressed 15 replication of influenza viruses, respiratory syncytial virus, human metapneumovirus, and severe acute respiratory syndrome coronavirus (SARS-CoV-1).(33) It has been proposed that IFN- $\lambda$ exerts much of its antiviral activity in vivo via stimulatory interactions with immune cells, rather than through induction of the antiviral cell state.(34) Importantly, IFN- $\lambda$ has been found to robustly restrict SARS-CoV-2 replication in primary human bronchial epithelial cells(35), primary human airway epithelial cultures(36), and primary human intestinal epithelial cells(37). Collectively, these studies suggest multifaceted mechanisms by which neutralizing IFN- $\lambda$ autoantibodies may exacerbate SARS-CoV-2 infections.

Casanova, et al. did not detect any type III IFN neutralizing antibodies among 101 individuals with type I IFN autoantibodies tested.(17) In our study, one of the three IFN- $\alpha$ 
autoreactive individuals (P2, a 22-year-old male) also harbored autoantibodies that neutralized IFN- $\lambda 3$. It is possible that this co-reactivity is extremely rare and thus not represented in the Casanova cohort. Alternatively, it is possible that the differing assay conditions exhibit differing detection sensitivity. Whereas Casanova, et al. cultured A549 cells with IFN- $\lambda 3$ at $50 \mathrm{ng} / \mathrm{ml}$

5 without plasma preincubation, we cultured A549 cells with IFN- $\lambda 3$ at $1 \mathrm{ng} / \mathrm{ml}$ after pre-incubation with plasma for one hour. Their readout of STAT3 phosphorylation may also provide different detection sensitivity compared to the upregulation of MX1 expression. A larger study is needed to determine the true frequency of these reactivities in severe COVID-19 patients and matched controls. Here, we report strongly neutralizing IFN- $\alpha$ and IFN- $\lambda 3$ autoantibodies in $3(5.5 \%)$ and $2(3.6 \%)$ individuals, respectively, of 55 patients with severe COVID-19. IFN- $\lambda 3$ autoantibodies were not detected via PhIP-Seq in a larger cohort of 423 healthy controls collected prior to the pandemic.

Type III interferons have been proposed as a therapeutic modality for SARS-CoV-2 infection, $(36,38-42)$ and there are currently three ongoing clinical trials to test pegylated IFN- $\lambda 1$

15 for efficacy in reducing morbidity and mortality associated with COVID-19 (ClinicalTrials.gov Identifiers: NCT04343976, NCT04534673, NCT04344600). One recently completed doubleblind, placebo-controlled trial, NCT04354259, reported a significant reduction by $2.42 \log$ copies per $\mathrm{ml}$ of SARS-CoV-2 at day 7 among mild to moderate COVID-19 patients in the outpatient setting ( $p=0 \cdot 0041)$.(43) Future studies will determine whether anti-IFN- $\lambda 3$ autoantibodies are preexisting or arise in response to SARS-CoV-2 infection, and how often they also cross-neutralize IFN- $\lambda 1$. Based on sequence alignment of IFN- $\lambda 1$ and IFN- $\lambda 3$ ( $\sim 29 \%$ homology, Fig. S4), however, cross-neutralization is expected to be rare, raising the possibility that patients with neutralizing IFN- $\lambda 3$ autoantibodies may especially derive benefit from pegylated IFN- $\lambda 1$ treatment. 


\section{Conclusions}

MIPSA is a new self-assembling protein display technology with key advantages over alternative approaches. It has properties that complement techniques like PhIP-Seq, and MIPSA ORFeome libraries can be conveniently screened in the same reactions with programmable phage

5 display libraries. The MIPSA protocol presented here requires cap-independent, cell-free translation, but future adaptations may overcome this limitation. Applications for MIPSA-based studies include protein-protein, protein-antibody, and protein-small molecule interaction studies, as well as unbiased analyses of post-translational modifications. Here we used MIPSA to detect known autoantibodies and to discover neutralizing IFN- $\lambda 3$ autoantibodies, among many other potentially pathogenic autoreactivities (Table S2), which may contribute to life-threatening COVID-19 pneumonia in a subset of at-risk individuals. 


\section{References}

1. H. B. Larman et al., Cytosolic 5 '-nucleotidase $1 \mathrm{~A}$ autoimmunity in sporadic inclusion body myositis. Annals of neurology 73, 408-418 (2013).

2. G. J. Xu et al., Viral immunology. Comprehensive serological profiling of human populations using a synthetic human virome. Science 348, aaao698 (2015).

3. $\quad$ E. Shrock et al., Viral epitope profiling of COVID-19 patients reveals cross-reactivity and correlates of severity. Science $\mathbf{3 7 0}$, (2020).

4. D. R. Monaco et al., Profiling serum antibodies with a pan allergen phage library identifies key wheat allergy epitopes. Nat Commun 12, 379 (2021).

10 5. S. F. Kingsmore, Multiplexed protein measurement: technologies and applications of protein and antibody arrays. Nat Rev Drug Discov 5, 310-320 (2006).

6. T. Kodadek, Protein microarrays: prospects and problems. Chem Biol 8, 105-115 (2001).

7. N. Ramachandran, E. Hainsworth, G. Demirkan, J. LaBaer, On-chip protein synthesis for making microarrays. Methods Mol Biol 328, 1-14 (2006).

15 8. S. Rungpragayphan, T. Yamane, H. Nakano, SIMPLEX: single-molecule PCR-linked in vitro expression: a novel method for high-throughput construction and screening of protein libraries. Methods Mol Biol 375, 79-94 (2007).

9. J. Zhu et al., Protein interaction discovery using parallel analysis of translated ORFs (PLATO). Nat Biotechnol 31, 331-334 (2013).

20 10. G. Liszczak, T. W. Muir, Nucleic Acid-Barcoding Technologies: Converting DNA Sequencing into a Broad-Spectrum Molecular Counter. Angew Chem Int Ed Engl 58, 4144-4162 (2019).

11. G. V. Los et al., HaloTag: a novel protein labeling technology for cell imaging and protein analysis. ACS Chem Biol 3, 373-382 (2008).

25 12. J. Yazaki et al., HaloTag-based conjugation of proteins to barcoding-oligonucleotides. Nucleic Acids Res 48, e8 (2020). 
13. F. Mohammad, R. Green, A. R. Buskirk, A systematically-revised ribosome profiling method for bacteria reveals pauses at single-codon resolution. Elife 8, (2019).

14. L. Gu et al., Multiplex single-molecule interaction profiling of DNA-barcoded proteins. Nature 515, 554-557 (2014).

5 15. X. Yang et al., A public genome-scale lentiviral expression library of human ORFs. Nat Methods 8, 659-661 (2011).

16. C. R. Consiglio et al., The Immunology of Multisystem Inflammatory Syndrome in Children with COVID-19. Cell 183, 968-981 e967 (2020).

17. P. Bastard et al., Autoantibodies against type I IFNs in patients with life-threatening COVID-19. Science 370, (2020).

18. Y. Zuo et al., Prothrombotic autoantibodies in serum from patients hospitalized with COVID-19. Sci Transl Med 12, (2020).

19. L. Casciola-Rosen et al., IgM autoantibodies recognizing ACE2 are associated with severe COVID-19. medRxiv, (2020).

15 20. M. C. Woodruff, R. P. Ramonell, F. E. Lee, I. Sanz, Broadly-targeted autoreactivity is common in severe SARS-CoV-2 Infection. medRxiv, (2020).

21. D. Wang et al., AAgAtlas 1.0: a human autoantigen database. Nucleic Acids Res 45, D769D776 (2017).

22. T. E. Lloyd et al., Cytosolic 5'-Nucleotidase 1A As a Target of Circulating Autoantibodies in Autoimmune Diseases. Arthritis Care Res (Hoboken) 68, 66-71 (2016).

23. E. Y. Wang et al., Diverse Functional Autoantibodies in Patients with COVID-19. medRxiv, (2020).

24. S. Gupta, S. Nakabo, J. Chu, S. Hasni, M. J. Kaplan, Association between anti-interferonalpha autoantibodies and COVID-19 in systemic lupus erythematosus. medRxiv, (2020).

25 25. G. J. Xu et al., Systematic autoantigen analysis identifies a distinct subtype of scleroderma with coincident cancer. Proc Natl Acad Sci US A, (2016). 
26. M. Stoeckius et al., Simultaneous epitope and transcriptome measurement in single cells. Nat Methods 14, 865-868 (2017).

27. I. Setliff et al., High-Throughput Mapping of B Cell Receptor Sequences to Antigen Specificity. Cell 179, 1636-1646 e1615 (2019).

28. S. K. Saka et al., Immuno-SABER enables highly multiplexed and amplified protein imaging in tissues. Nat Biotechnol 37, 1080-1090 (2019).

29. M. A. Jongsma, R. H. Litjens, Self-assembling protein arrays on DNA chips by autolabeling fusion proteins with a single DNA address. Proteomics 6, 2650-2655 (2006).

30. A. Gautier et al., An engineered protein tag for multiprotein labeling in living cells. Chem Biol 15, 128-136 (2008).

31. A. J. Samelson et al., Kinetic and structural comparison of a protein's cotranslational folding and refolding pathways. Sci Adv 4, eaas9098 (2018).

32. L. Tosi et al., Long-adapter single-strand oligonucleotide probes for the massively multiplexed cloning of kilobase genome regions. Nat Biomed Eng 1, (2017).

15 33. M. Mordstein et al., Lambda interferon renders epithelial cells of the respiratory and gastrointestinal tracts resistant to viral infections. $J$ Virol 84, 5670-5677 (2010).

34. N. Ank et al., Lambda interferon (IFN-lambda), a type III IFN, is induced by viruses and IFNs and displays potent antiviral activity against select virus infections in vivo. $J$ Virol 8o, 4501-4509 (2006).

20 35. I. Busnadiego et al., Antiviral Activity of Type I, II, and III Interferons Counterbalances ACE2 Inducibility and Restricts SARS-CoV-2. mBio 11, (2020).

36. A. Vanderheiden et al., Type I and Type III Interferons Restrict SARS-CoV-2 Infection of Human Airway Epithelial Cultures. J Virol 94, (2020).

37. M. L. Stanifer et al., Critical Role of Type III Interferon in Controlling SARS-CoV-2 Infection in Human Intestinal Epithelial Cells. Cell Rep 32, 107863 (2020).

38. I. E. Galani et al., Untuned antiviral immunity in COVID-19 revealed by temporal type I/III interferon patterns and flu comparison. Nat Immunol 22, 32-40 (2021). 
39. U. Felgenhauer et al., Inhibition of SARS-CoV-2 by type I and type III interferons. $J$ Biol Chem 295, 13958-13964 (2020).

40. T. R. O'Brien et al., Weak Induction of Interferon Expression by Severe Acute Respiratory Syndrome Coronavirus 2 Supports Clinical Trials of Interferon-lambda to Treat Early Coronavirus Disease 2019. Clin Infect Dis 71, 1410-1412 (2020).

41. E. Andreakos, S. Tsiodras, COVID-19: lambda interferon against viral load and hyperinflammation. EMBO Mol Med 12, e12465 (2020).

42. L. Prokunina-Olsson et al., COVID-19 and emerging viral infections: The case for interferon lambda. $J$ Exp Med 217, (2020). phase 2, placebo-controlled randomised trial. Lancet Respir Med, (2021).

44. D. Mohan et al., Publisher Correction: PhIP-Seq characterization of serum antibodies using oligonucleotide-encoded peptidomes. Nature protocols 14, 2596 (2019).

45. C. Tuckey, H. Asahara, Y. Zhou, S. Chong, Protein synthesis using a reconstituted cell-free system. Curr Protoc Mol Biol 108, 1631 11-22 (2014).

46. S. L. Klein et al., Sex, age, and hospitalization drive antibody responses in a COVID-19 convalescent plasma donor population. J Clin Invest 130, 6141-6150 (2020).

47. Correction: Patient Trajectories Among Persons Hospitalized for COVID-19. Ann Intern Med 174, 144 (2021). DA, Lieberman MR, Bigajer E, Stok D, Frank E, Silverberg JI, SARS-CoV-2 Seroprevalence and Symptom Onset in Culturally-Linked Orthodox Jewish Communities Across Multiple Regions in the United States. JAMA Open Network In Press, (2021).

49. M. R. Rose, E. I. W. Group, 188th ENMC International Workshop: Inclusion Body 1055 (2013). 
50. Z. Wei, W. Zhang, H. Fang, Y. Li, X. Wang, esATAC: an easy-to-use systematic pipeline for ATAC-seq data analysis. Bioinformatics 34, 2664-2665 (2018).

51. M. D. Robinson, D. J. McCarthy, G. K. Smyth, edgeR: a Bioconductor package for differential expression analysis of digital gene expression data. Bioinformatics 26, 139$140(2010)$.

52. brandonsie.github.io/epitopefindr/

\section{Acknowledgements}

We would like to thank Steve Elledge for generously providing the human ORFeome

Catipovic, Allen Buskirk, Tim O'Donnell, Priya Duggal and Janet Markle for helpful discussions.

We also thank Jodie Franklin and the Johns Hopkins Synthesis and Sequencing Core for HPLC purification of the HaloLigand conjugated RT-primer, as well as Linda Orzolek and Haiping Hao of the Johns Hopkins Transcriptomics and Deep Sequencing Core Facility.

The severe COVID-19 specimens utilized for this publication were part of the Johns Hopkins Biospecimen Repository, which is based on the contribution of many patients, research teams, and clinicians. Thanks to members of the NIH Vaccine Research Center for pre-pandemic sample collection: Barney Graham, Laura Novick, Joseph Casazza, Julie Ledgerwood, Uzma Sarwar, LeeJah Chang, Cynthia Starr Hendel, Lasonji Holman, Sarah Plummer, Pam Costner,

Galina Yamshchikov, Iris Pittman, Pernell Williams.

All figures were created with BioRender.com

\section{Funding:}


National Institute of General Medical Sciences grant R01GM127533 (HBL, BP)

National Heart, Lung, and Blood Institute of the National Institutes of Health grant K23HL151826 (EMB)

Sjogren's Foundation and the Jerome L. Greene Foundation grants (ANB, HBL)

Vaccine Research Center, National Institute of Allergy and Infectious Diseases, National

Institutes of Health intramural research program (MR)

\section{Author contributions:}

Conceptualization and experimental design: JJC, HBL

Formal analysis: JJC, JG, PS, DRM, XAZ, WRM, YD, SJ, HBL

Funding acquisition: BP, HBL

Resources: ANB, MR, EMB, AART, IZ, JIS, AZR, ALC, TL, ALM

Visualization: JJC, JG, PS, DRM, XAZ, WRM

Writing: JJC, JG, PS, DRM, XAZ, HJT, AW, WRM, YD, SJ, LT, BP, ANB, MR, EMB, AART, IZ, JIS, AZR, ALC, TL, ALM, HBL

\section{Competing interests:}

HBL, JJC, JG, and PS are inventors on a patent application filed by Johns Hopkins University that and is an advisor to CDI Laboratories and TScan Therapeutics. 


\section{Supplementary Materials}

\section{Material and Methods}

MIPSA Destination vector construction

The MIPSA vector was constructed using the Gateway pDEST15 vector as a backbone. A gBlock fragment (Integrated DNA Technologies) encoding the RBS, Kozak sequence, N-terminal HaloTag fusion protein, and FLAG tag, followed by an attR1 sequence was cloned into the parent plasmid. A stop codon and 150 bp poly(A) sequence was also added after attR2 site.

A 41 nt barcode oligo was generated within a gBlock Gene Fragment (Integrated DNA Technologies) with alternating mixed bases $(\mathrm{S}: \mathrm{G} / \mathrm{C} ; \mathrm{W}: \mathrm{A} / \mathrm{T})$ to produce the following sequence: $(\mathrm{SW})_{18}$-AGGGA-(SW)18. The sequences flanking the degenerate barcode incorporated the standard PhIP-Seq PCR1 and PCR2 primer binding sites.(44) 18 ng of the starting UCI library was used to run 40 cycles of PCR to amplify the library and incorporate BgIII and PspxI restriction sites. The MIPSA vector and amplified UCI library were then digested with the restriction enzymes overnight, column purified, and ligated at 1:5 vector-to-insert ratio. The ligated MIPSA vector was used to transform electrocompetent One Shot $\operatorname{ccdB} 2 \mathrm{~T}^{\mathrm{R}}$ cells (Thermo Fisher Scientific). 6 transformation reactions yielded $\sim 800,000$ colonies to produce the pDEST-MIPSA UCI library.

\section{Human ORFeome recombination into the pDEST-MIPSA UCI plasmid library}

$150 \mathrm{ng}$ of each pENTR-hORFeome subpool (L1-L5) was individually combined with 150 ng of the pDEST-MIPSA UCI library plasmid and $2 \mu \mathrm{l}$ of Gateway LR Clonase II mix (Life Technologies) for a total reaction volume of $10 \mu \mathrm{l}$. The reaction was incubated overnight at $25^{\circ} \mathrm{C}$. 
The entire reaction was transformed into $50 \mu$ of One Shot OmniMAX $2 \mathrm{~T}^{\mathrm{R}}$ chemical competent E. coli (Life Technologies). In aggregate, the transformations yielded $\sim 120,000$ colonies, which is $\sim 10$-fold the complexity of the hORFeome v8.1. Colonies were collected and pooled by scraping, followed by purification of the barcoded pDEST-MIPSA-hORFeome plasmid DNA (human $5 \quad$ ORFeome MIPSA library) using the Qiagen Plasmid Midi Kit (Qiagen).

\section{HaloLigand conjugation to RT oligo and HPLC purification}

$100 \mu \mathrm{g}$ of a 5' amine modified oligo HL-32_ad (Table S3) was incubated with $75 \mu 1$ (17.85 $\mu \mathrm{g} / \mu \mathrm{l})$ of the HaloTag Succinimidyl Ester (O2) (Promega Corporation), the HaloLigand, in $0.1 \mathrm{M}$ sodium borate buffer for 6 hours at room temperature following $\mathrm{Gu}$, et al.(14) $3 \mathrm{M} \mathrm{NaCl}$ and icecold ethanol was added at $10 \%(\mathrm{v} / \mathrm{v})$ and $250 \%(\mathrm{v} / \mathrm{v})$, respectively, to the labeling reaction and incubated overnight at $-80^{\circ} \mathrm{C}$. The reaction was centrifuged for 30 minutes at $12,000 \mathrm{x}$. The pellet was rinsed once in ice-cold $70 \%$ ethanol and air-dried for 10 minutes.

HaloLigand-conjugated RT primer was HPLC purified using a Brownlee Aquapore RP-

$153007 \mathrm{u}, 100 \times 4.6 \mathrm{~mm}$ column (Perkin Elmer) using a two-buffer gradient of $0-70 \% \mathrm{CH} 3 \mathrm{CN} / \mathrm{MeCN}$ (100 mM triethylamine acetate to acetonitrile) over 70 minutes. Fractions corresponding to labeled oligo were collected and lyophilized (Fig. S1). Oligos were resuspended at $1 \mu \mathrm{M}(15.4 \mathrm{ng} / \mu \mathrm{l})$ and stored at $-80^{\circ} \mathrm{C}$.

The human ORFeome MIPSA library plasmid $(4 \mu \mathrm{g})$ was linearized with the I-SceI restriction endonuclease (New England Biolabs) overnight. The product was column-purified with the NucleoSpin Gel and PCR Clean Up kit (Macherey-Nagel). A $40 \mu$ in vitro transcription reaction using the HiScribe T7 High Yield RNA Synthesis Kit (New England Biolabs) was utilized 
to transcribe $1 \mu \mathrm{g}$ of the purified, linearized pDEST-MIPSA plasmid library. The product was diluted with $60 \mu \mathrm{l}$ molecular biology grade water, and $1 \mu \mathrm{l}$ of DNAse I was added. The reaction was incubated for another 15 minutes at $37^{\circ} \mathrm{C}$. Then $50 \mu \mathrm{l}$ of $1 \mathrm{M} \mathrm{LiCl}$ was added to the solution and incubated at $-80^{\circ} \mathrm{C}$ overnight. A centrifuge was cooled to $4{ }^{\circ} \mathrm{C}$, and the RNA was spun at maximum speed for 30 minutes. The supernatant was removed, and the RNA pellet washed with $70 \%$ ethanol. The sample was spun down at $4{ }^{\circ} \mathrm{C}$ for another 10 minutes, and the $70 \%$ ethanol removed. The pellet was dried at room temperature for 15 minutes, and subsequently resuspended in $100 \mu \mathrm{l}$ water. To preserve the sample, $1 \mu \mathrm{l}$ of $40 \mathrm{U} / \mu \mathrm{l}$ RNAseOUT Recombinant Ribonuclease Inhibitor (Life Technologies) was added.

\section{MIPSA library IVT-RNA reverse transcription and translation}

A reverse transcription reaction was prepared using SuperScript IV First-Strand Synthesis

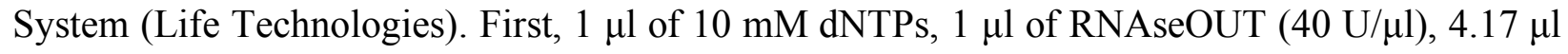
of the RNA library $(1.5 \mu \mathrm{M})$, and $7.83 \mu$ of the HaloLigand-conjugated RT primer $(1 \mu \mathrm{M}$, Table

S3) was combined for a single $14 \mu \mathrm{l}$ reaction and incubated at $65^{\circ} \mathrm{C}$ for 5 minutes followed by a 2-minute incubation on ice. $4 \mu \mathrm{l}$ of $5 \mathrm{X}$ RT buffer, $1 \mu \mathrm{l}$ of $0.1 \mathrm{M}$ DTT, and $1 \mu 1$ of SuperScript IV RT Enzyme $(200 \mathrm{U} / \mu \mathrm{l})$ was added to the $14 \mu \mathrm{l}$ reaction on ice and incubated for 20 minutes at $42^{\circ} \mathrm{C}$. A single $20 \mu \mathrm{RT}$ reaction received $36 \mu \mathrm{l}$ of RNAClean XP beads (Beckman Coulter) and was incubated at room temperature for 10 minutes. The beads were collected by magnet and washed five times with $70 \%$ ethanol. The beads were air-dried for 10 minutes at room temperature and resuspended in $7 \mu \mathrm{l}$ of $5 \mathrm{mM}$ Tris- $\mathrm{HCl}, \mathrm{pH}$ 8.5. The product was analyzed with spectrophotometry to measure the RNA yield. A translation reaction was set up on ice using the PURExpress $\Delta$ Ribosome Kit (New England Biolabs).(45) The reaction was modified such that the final concentration of ribosomes was $0.3 \mu \mathrm{M}$. For each $10 \mu \mathrm{l}$ translation reaction, $4.57 \mu$ of the 
RT reaction was added to $4 \mu \mathrm{l}$ Solution A, $1.2 \mu 1$ Factor Mix, and $0.23 \mu 1$ ribosomes $(13.3 \mu \mathrm{M})$.

This reaction was incubated at $37^{\circ} \mathrm{C}$ for two hours, diluted to a total volume of $45 \mu 1$ with $35 \mu 1$ $1 \mathrm{X}$ PBS, and used immediately or stored at $-80^{\circ} \mathrm{C}$ after addition of glycerol to a final concentration of $25 \%(\mathrm{v} / \mathrm{v})$.

Immunoprecipitation of the translated MIPSA hORFeome library

$5 \mu \mathrm{l}$ of plasma, diluted 1:100 in PBS, is mixed with the $45 \mu \mathrm{l}$ of diluted MIPSA library translation reaction (see above) and incubated overnight at $4^{\circ} \mathrm{C}$ with gentle agitation. For each IP, a mixture of $5 \mu \mathrm{l}$ of Protein A Dynabeads and $5 \mu \mathrm{l}$ of Protein G Dynabeads (Life Technologies) in 1X PBS at their original volume, and added to each IP. The antibody capture proceeded for 4 hours at $4^{\circ} \mathrm{C}$. Beads were collected on a magnet and washed 3 times in $1 \mathrm{X}$ PBS, changing tubes or plates between washes. The beads were then collected and resuspended in a $20 \mu \mathrm{l}$ PCR master mix containing the T7-Pep2_PCR1_F forward and the T7-Pep2_PCR1_R+ad_min reverse primers

15 (Table S3) and Herculase-II (Agilent). PCR cycling was as follows: an initial denaturing and enzyme activation step at $95^{\circ} \mathrm{C}$ for $2 \mathrm{~min}$, followed by 45 cycles of: $95^{\circ} \mathrm{C}$ for $20 \mathrm{~s}, 58^{\circ} \mathrm{C}$ for $30 \mathrm{~s}$, and $72^{\circ} \mathrm{C}$ for $30 \mathrm{~s}$. The final extension step was performed at $72^{\circ} \mathrm{C}$ for 3 minutes. Two microliters of the amplification product were used as input to a $20 \mu \mathrm{l}$ dual-indexing PCR reaction for 10 cycles with the PhIP_PCR2_F forward and the Ad_min_BCX_P7 reverse primers. PCR cycling was as follows: an initial denaturing step at $95^{\circ} \mathrm{C}$ for $2 \mathrm{~min}$, followed by 10 cycles of: $95^{\circ} \mathrm{C}$ for $20 \mathrm{~s}, 58^{\circ} \mathrm{C}$ for $30 \mathrm{~s}$, and $72^{\circ} \mathrm{C}$ for $30 \mathrm{~s}$. The final extension step was performed at $72^{\circ} \mathrm{C}$ for $3 \mathrm{~min}$. i5/i 7 indexed libraries were pooled and column purified (NucleoSpin columns, Takara). Libraries were sequenced on an Illumina NextSeq 500 using a 1x50 nt SE or 1x75 nt SE protocol. 
MIPSA_i5_NextSeq_SP and Standard_i7_SP primers were used for i5/i7 sequencing (Table S3)

The output was demultiplexed using i5 and i7 without allowing any mismatches.

For quantification of MIPSA experiments by qPCR, the PCR1 product (above) was analyzed as follows. A $4.6 \mu$ of 1:1,000 dilution of the PCR1 reaction was added to $5 \mu$ l of Brilliant

$5 \quad$ III Ultra Fast $2 X$ SYBR Green Mix (Agilent), $0.2 \mu \mathrm{l}$ of $2 \mu \mathrm{M}$ reference dye and $0.2 \mu 1$ of $10 \mu \mathrm{M}$ forward and reverse primer mix (specific to the target UCI). PCR cycling was as follows: an initial denaturing step at $95^{\circ} \mathrm{C}$ for $2 \mathrm{~min}$, followed by 45 cycles of: $95^{\circ} \mathrm{C}$ for $20 \mathrm{~s}, 60^{\circ} \mathrm{C}$ for 30 . Following completion of thermocycling, amplified products were subjected to melt-curve analysis. The qPCR primers for MIPSA immunoprecipitation experiments were: BT2_F and BT2_R for TRIM21, BG4_F and BG4_R for GAPDH, and NT5C1A_F and NT5C1A_R for NT5C1A (Table S3).

\section{Plasma Samples}

All samples were collected from subjects that met protocol eligibility criteria, as described below. All of the studies protected the rights and privacy of the study participants and were approved by their respective Intuitional Review Boards for original sample collection and subsequent analyses.

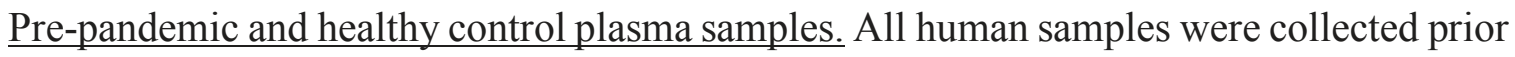
to 2017 at the National Institutes of Health (NIH) Clinical Center under the Vaccine Research compliance with NIAID IRB approved procedures.

COVID-19 Convalescent Plasma (CCP) from non-hospitalized patients. Eligible nonhospitalized CCP donors were contacted by study personnel, as previously described.(46) All 
donors were at least 18 years old and had a confirmed diagnosis of SARS-CoV-2 by detection of RNA in a nasopharyngeal swab sample. Basic demographic information (age, sex, hospitalization with COVID-19) was obtained from each donor; initial diagnosis of SARS-CoV-2 and the date of diagnosis were confirmed by medical chart review.

Severe COVID-19 plasma samples. The study cohort was defined as inpatients who had: 1) a confirmed RNA diagnosis of COVID-19 from a nasopharyngeal swab sample; 2) survival to death or discharge; and 3) remnant specimens in the Johns Hopkins COVID-19 Remnant Specimen Biorepository, an opportunity sample that includes 59\% of Johns Hopkins Hospital COVID-19 patients and $66 \%$ of patients with length of stay $\geq 3$ days. $(47,48)$ Patient outcomes were defined by the World Health Organization (WHO) COVID-19 disease severity scale. Samples from severe COVID-19 patients that were included in this study were obtained from 17 patients who died, 13 who recovered after being ventilated, 22 who required oxygen to recover, and 3 who recovered without supplementary oxygen. This study was approved by the JHU Institutional Review Board (IRB00248332, IRB00273516), with a waiver of consent because all specimens and clinical data

15 were de-identified by the Core for Clinical Research Data Acquisition of the Johns Hopkins Institute for Clinical and Translational Research; the study team had no access to identifiable patient data.

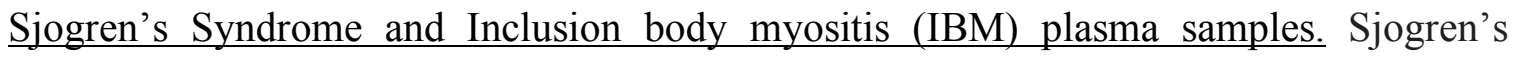
syndrome samples were collected under protocol NA_00013201. All patients were $>18$ years old and gave informed consent. IBM patient samples were collected under protocol IRB00235256. All patients met ENMC 2011 diagnostic criteria(49) and provided informed consent. 


\section{Immunoblot analysis}

Laemmli buffer containing 5\% $\beta$-ME was added to samples, boiled for $5 \mathrm{~min}$, and analyzed on NuPage 4-12\% Bis-Tris polyacrylamide gels (Life Technologies). Following transfer to PVDF membranes, blots were blocked in $20 \mathrm{mM}$ Tris-buffered saline, $\mathrm{pH} 7.6$, containing $0.1 \%$ Tween

$520(\mathrm{TBST})$ and 5\% (wt/vol) non-fat dry milk for 30 minutes at room temperature. Blots were subsequently incubated overnight at $4^{\circ} \mathrm{C}$ with primary anti-FLAG antibody (\#F3165, MilliporeSigma) at 1:2,000 (v/v), followed by a 4-hour incubation at room temperature in antimouse IgG, HRP-linked secondary antibody (\#7076, Cell Signaling) at 1:4,000 (v/v).

The Nextera XT DNA Library Preparation kit (Illumina) was used for tagmentation of 150 ng of the pDEST-MIPSA hORFeome plasmid library to yield the optimal size distribution centered around $1.5 \mathrm{~kb}$. Tagmented libraries were amplified using Herculase-II (Agilent) with T7Pep2_PCR1_F forward and Nextera Index 1 Read primer. PCR cycling was as follows: an initial denaturing step at $95^{\circ} \mathrm{C}$ for 2 minutes, followed by 30 cycles of: $95^{\circ} \mathrm{C}$ for $20 \mathrm{~s}, 53.5^{\circ} \mathrm{C}$ for $30 \mathrm{~s}$, $72^{\circ} \mathrm{C}$ for $30 \mathrm{~s}$. A final extension step was performed at $72^{\circ} \mathrm{C}$ for 3 minutes. PCR reactions were run on a $1 \%$ agarose gel followed by excision of $\sim 1.5 \mathrm{~kb}$ products and purification using the NucleoSpin Gel and PCR Clean-up columns (Macherey-Nagel). The purified product was then amplified for another 10 cycles with PhIP_PCR2_F forward and P7.2 reverse primers (see Table S3 for list of primer sequences). The product was gel-purified and sequenced on a MiSeq (Illumina) using the T7-Pep2.2_SP_subA primer for read 1 and the MISEQ_MIPSA_R2 primer for read 2. Read 1 was 60 bp long to capture the UCIs. The first index read, I1, was substituted with a 50 bp read into the ORF. I2 was used to identify the i5 index for sample demultiplexing. 
The hORFeome v8.1 DNA sequences were truncated to the first $50 \mathrm{nt}$, and the ORF names corresponding to non-unique sequences were concatenated with a "|" delimiter. The demultiplexed output of the $50 \mathrm{nt}$ R2 (ORF) read from an Illumina MiSeq was aligned to the truncated human ORFeome v8.1 library using the Rbowtie2 package with the following parameters: options = "-a -very-sensitive-local".(50) The unique FASTQ identifiers were then used to extract corresponding sequences from the $60 \mathrm{bp} \mathrm{R1}$ (UCI) read. Those sequences were then truncated using the 3' anchor ACGATA, and sequences that did not have the anchor were removed. Additionally, any truncated R1 sequences that had fewer than 18 nucleotides were removed. The ORF sequences that still had a corresponding UCI post-filtering were retained using the FASTQ identifier. The names of ORFs that had the same UCI were concatenated with a "\&" delimiter, and this final dictionary was used to generate a FASTA alignment file composed of ORF names and UCI sequences.

\section{Informatic analysis of MIPSA sequencing data}

Illumina output FASTQ files were truncated using the constant ACGAT anchor sequence

15 following all UCI sequences. Next, perfect match alignment was used to map the truncated sequences to their linked ORFs via the UCI-ORF lookup dictionary. A counts matrix is constructed, in which rows correspond to individual UCIs and columns correspond to samples. We next used the edgeR software package(51) which, using a negative binomial model, compares the signal detected in each sample against a set of negative control ("mock") IPs that were performed without plasma, to return a maximum likelihood fold-change estimate and a test statistic for each UCI in every sample, thus creating fold-change and $-\log 10(p-v a l u e)$ matrices. Significantly enriched UCIs ("hits") required a read count of at least 15, a p-value less than 0.001 , and a fold change of at least 3 . Hits fold-change matrices report the fold-change value for "hits" and report a "1" for UCIs that are not hits. 


\section{Protein sequence similarity}

To evaluate sequence homology among proteins in the hORFeome v8.1 library, a blastp alignment was used to compare each protein sequence against all other library members

5 (parameters: “-outfmt 6 -evalue 100 -max_hsps 1 -soft_masking false -word_size 7 max_target_seqs 100000”). To evaluate sequence homology among reactive peptides in the human 90-aa phage display library, the epitopefindr(52) software was employed.

\section{Phage ImmunoPrecipitation Sequencing (PhIP-Seq) analyses} immunoprecipitated using protein $A$ and protein $G$ coated magnetic beads. A set of 6-8 mock immunoprecipitations (no plasma input) were run on each 96 well plate. Magnetic beads were resuspended in PCR master mix and subjected to thermocycling. A second PCR reaction was 500 instrument using a $1 \times 50 \mathrm{nt}$ SE or $1 \times 75 \mathrm{nt}$ SE protocol. PhIP-Seq with the human library was used to characterize autoantibodies in a collection of plasma from healthy controls. For fair comparison to the severe COVID-19 cohort, we first determined the minimum sequencing depth that would have been required to detect the IFN- $\lambda 3$ reactivity in both of the positive individuals.

We then only considered the 423 data sets from the healthy cohort with sequencing depth greater than this minimum threshold. None of these 423 individuals were found to be reactive to any peptide from IFN- $\lambda 3$. 


\section{Type I/III interferon neutralization assay}

IFN- $\alpha 2$ (catalog no. 11100-1) and IFN- $\lambda 3$ (catalog no. 5259-IL-025) were purchased from

R\&D Systems. $20 \mu 1$ of plasma was incubated for 1 hour at room temperature with either $100 \mathrm{U} / \mathrm{ml}$

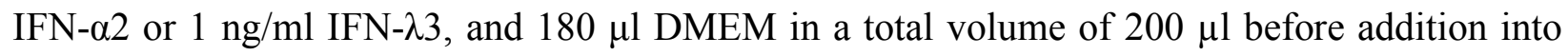

$5 \quad 7.5 \times 10^{4}$ A549 cells in 48-well tissue culture plates. After 4-hour incubation, the cells were washed with 1x PBS and cellular mRNA was extracted and purified using RNeasy Plus Mini Kit (Qiagen). $600 \mathrm{ng}$ of extracted mRNA was reverse transcribed using the SuperScript III First-Strand Synthesis System (Life Technologies) and diluted 10-fold for qPCR analysis on a QuantStudio 6 Flex System (Applied Biosystems). PCR consisted of $95^{\circ} \mathrm{C}$ for 3 minutes, followed by 45 cycles of the GAPDH expression. The qPCR primers for GAPDH and MX1 were obtained from Integrated DNA Technologies (Table S3). 
Fig. $\mathbf{~ 1 ~}$

A

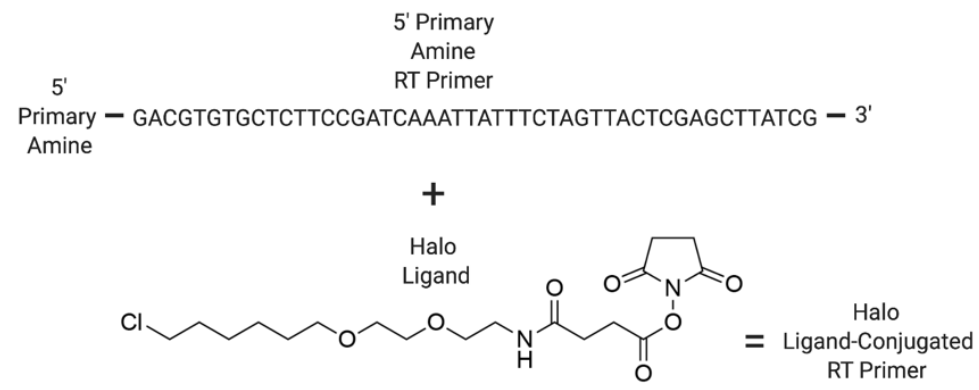

B

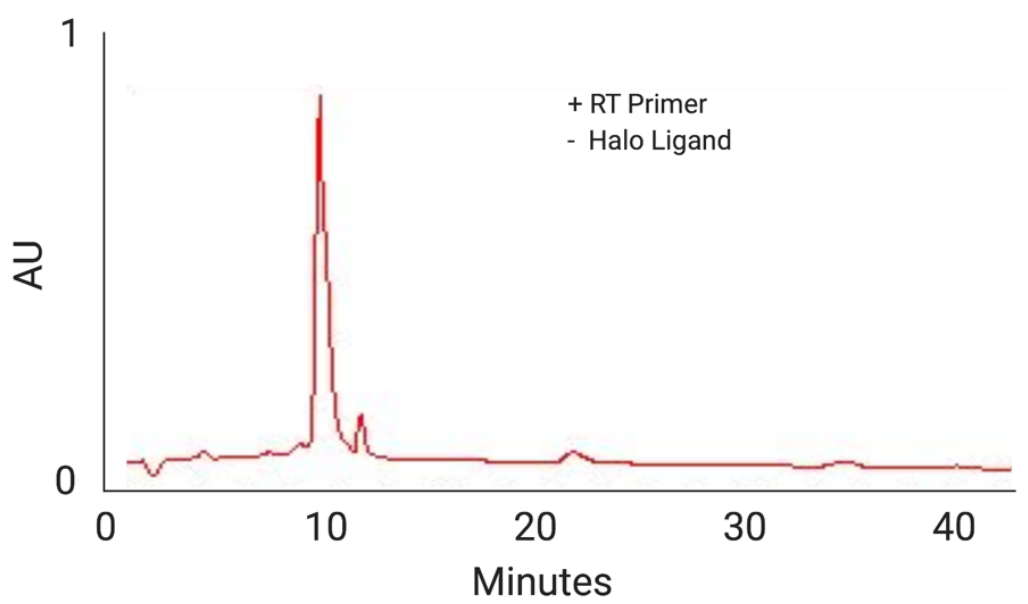

C

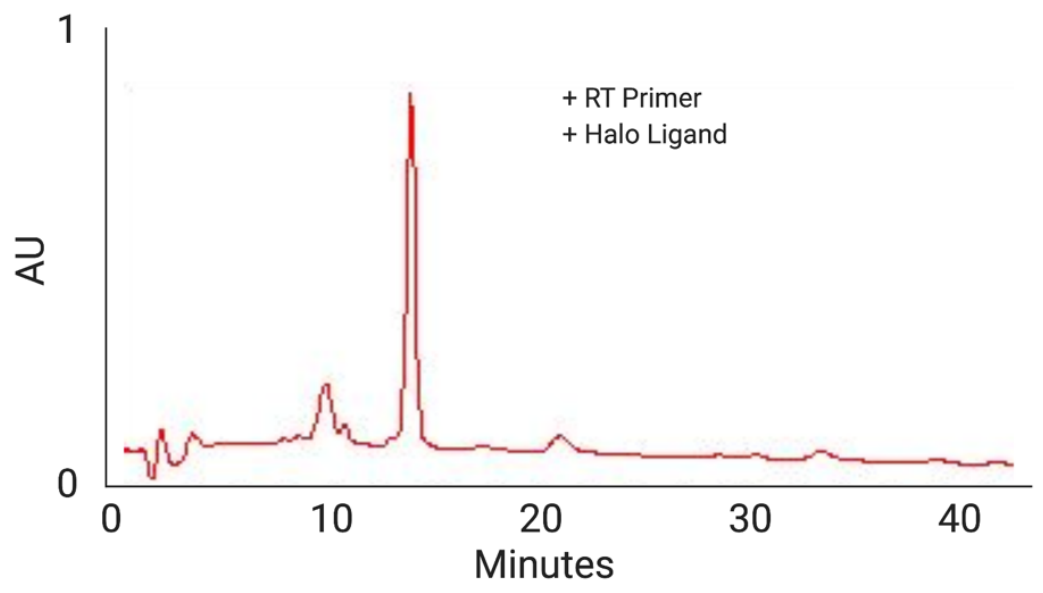

Fig. S1. HaloLigand conjugation to the reverse transcription primer. (A) On the top is the oligonucleotide reverse transcription (RT) primer sequence modified with a 5' primary amine. Below is the HaloLigand with a reactive succinimidyl ester group, separated by one ethylene glycol moiety (O2). The succinimidyl ester reacts with the primary amine to form an amide-bond between the RT primer and the HaloLigand, resulting in the HaloLigand-conjugated RT primer. (B) HPLC chromatogram of the RT primer without the HaloLigand modification. (C) HPLC chromatogram of the RT primer with the HaloLigand modification after purification. The conjugated product is eluted off the column later due to its decreased hydrophobicity conferred by the modification. 

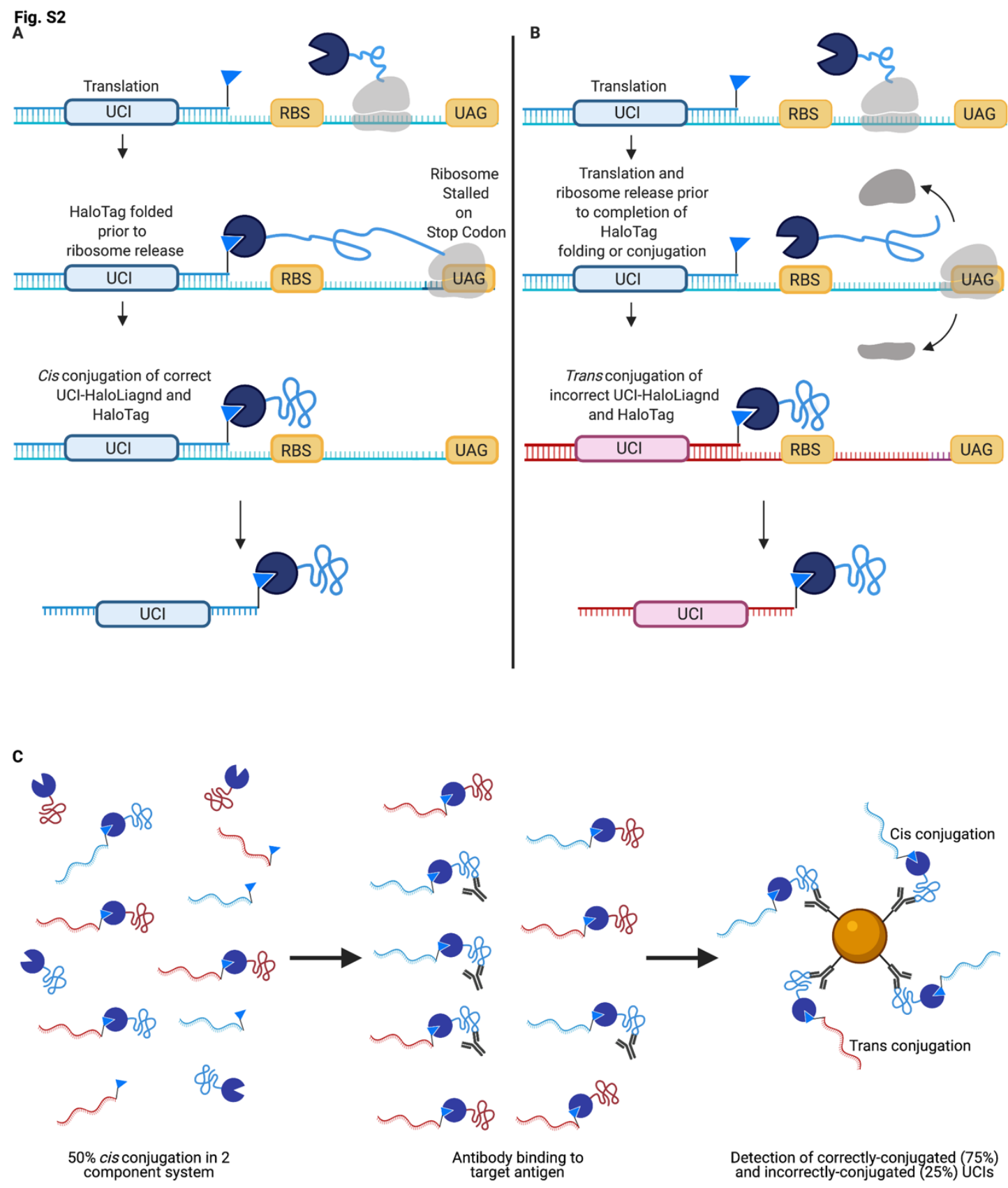

Fig. S2. Cis versus trans UCI-ORF associations. Schematic of cis (A) versus trans (B) UCI-ORF conjugation during translation of a MIPSA IVT-RNA library. (C) Left panel: 50\% cis conjugation composed of the correct protein-UCI associations (e.g. blue UCI with blue protein). Unconjugated proteins randomly associate with unconjugated UCIs (in trans). Middle panel: antibodies bind their target antigen. Right panel: the ratio of correctly to incorrectly IPed UCIs in this two-species experiment is 3:1 (75\%:25\%), similar to what was observed experimentally (Fig. 2A). 
Fig. S3

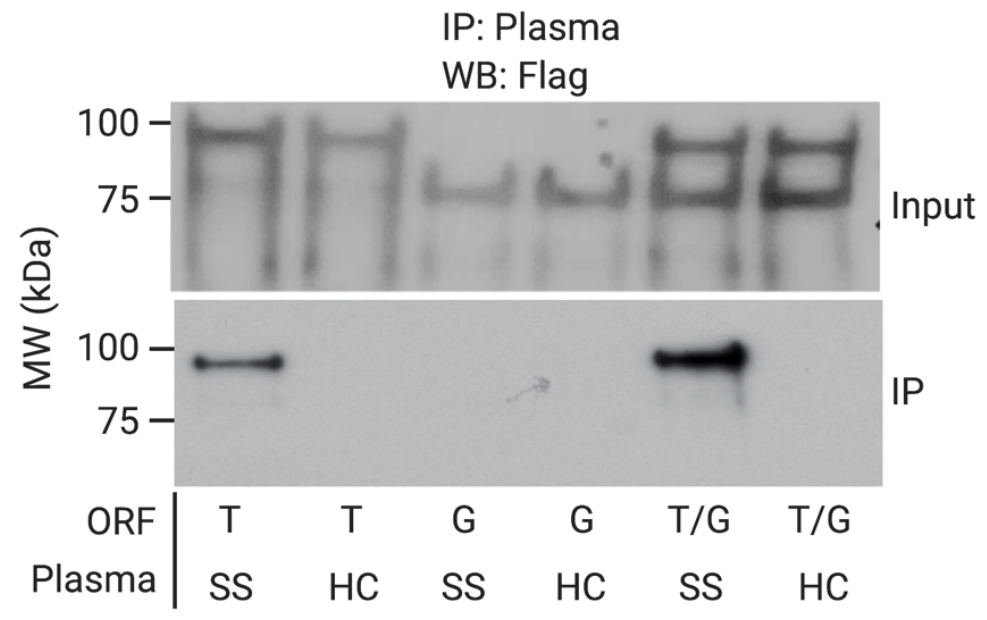

Fig. S3. Two-plex translation and IP of TRIM21 and GAPDH. TRIM21 (T) and GAPDH (G) IVT-RNA-cDNA were translated either separately or together and then subjected to IP with 5 healthy control (HC) or Sjogren's Syndrome (SS) plasma. Analysis was by immunoblotting with the M2 antibody that recognizes the common FLAG epitope tag that links the HaloTag to the protein. 
Fig. 54

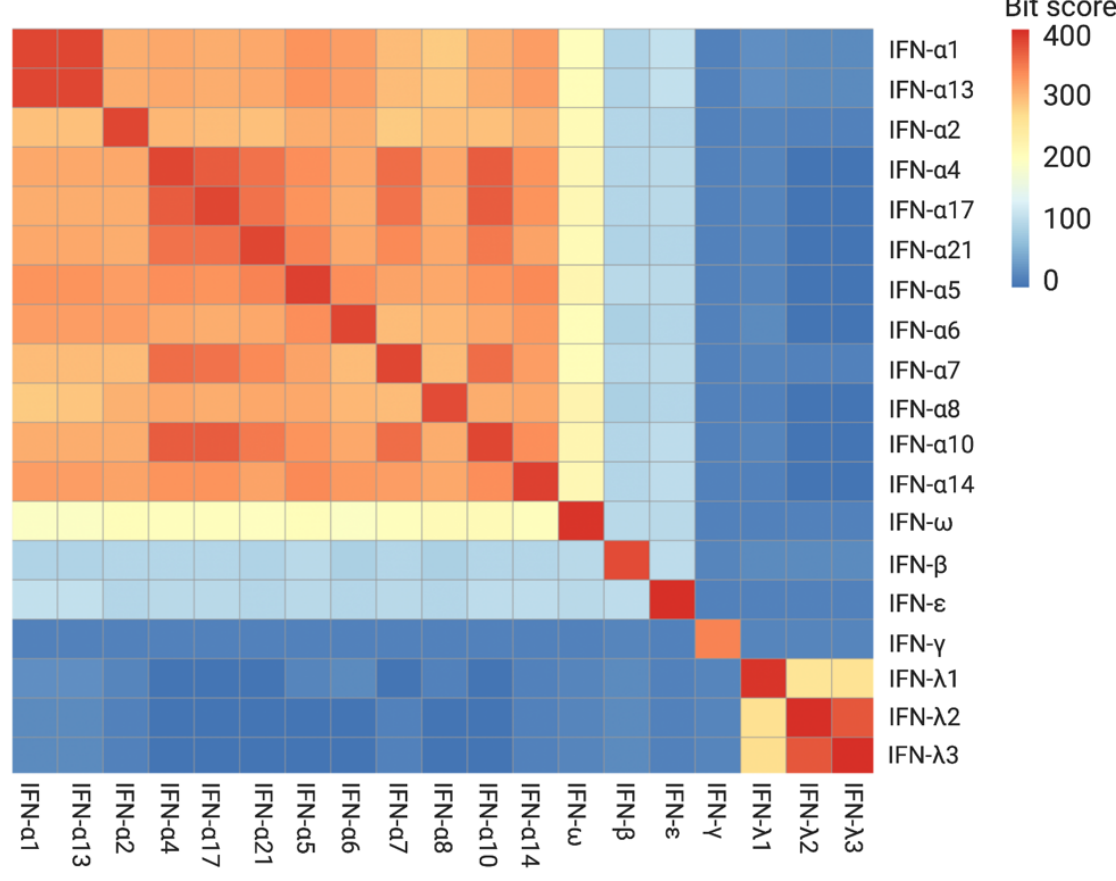

Fig. S4. Sequence homology of interferons. Pairwise blastp alignment bitscore matrix for all interferon (IFN) proteins shown in Fig. 5D. 


\begin{tabular}{|c|c|c|c|c|c|c|c|}
\hline \multicolumn{8}{|c|}{ Study Population } \\
\hline \multicolumn{2}{|c|}{ Group } & \multirow{2}{*}{$\begin{array}{c}\# \\
17\end{array}$} & \multirow{2}{*}{$\frac{\text { Age }}{67(27,87)}$} & \multirow{2}{*}{$\frac{\text { Sex }}{\mathrm{F}: 8, \mathrm{M}: 9}$} & \multirow{2}{*}{$\begin{array}{c}\text { Black } \\
9\end{array}$} & \multirow{2}{*}{$\begin{array}{c}\text { White } \\
4\end{array}$} & \multirow{2}{*}{$\begin{array}{c}\text { Other } \\
4\end{array}$} \\
\hline \multirow{4}{*}{$\begin{array}{c}\text { Severe } \\
\text { COVID-19 }\end{array}$} & Died & & & & & & \\
\hline & Ventilated & 13 & $67(27,82)$ & $\mathrm{F}: 9, \mathrm{M}: 4$ & 4 & 4 & 5 \\
\hline & Got O2 & 22 & $52(27,82)$ & $F: 9, M: 13$ & 8 & 8 & 6 \\
\hline & No $\mathrm{O} 2$ & 3 & $46(22,49)$ & $\mathrm{F}: 0, \mathrm{M}: 3$ & 0 & 3 & 0 \\
\hline \multirow{2}{*}{$\begin{array}{l}\text { COVID-19 } \\
\text { Controls }\end{array}$} & Mild/Mod & 10 & $35(19,55)$ & $\mathrm{F}: 6, \mathrm{M}: 4$ & 0 & 8 & 2 \\
\hline & Healthy Control & 10 & $41.5(22,66)$ & $\mathrm{F}: 3, \mathrm{M}: 7$ & 3 & 5 & 2 \\
\hline \multirow{2}{*}{ Myositis } & Inclusion Body & 10 & $53.9(43.6,60.6)$ & $\mathrm{F}: 7, \mathrm{M}: 3$ & 1 & 7 & 2 \\
\hline & Healthy Control & 10 & $36.5(20,60)$ & $\mathrm{F}: 5, \mathrm{M}: 5$ & 2 & 8 & 0 \\
\hline
\end{tabular}

Table S1. Severe COVID-19 patients and control study participants. Individuals' ages were provided to investigators as intervals to protect identities of study participants. 
bioRxiv preprint doi: https://doi.org/10.1101/2021.03.02.432977; this version posted March 3, 2021. The copyright holder for this preprint (which was not certified by peer review) is the author/funder, who has granted bioRxiv a license to display the preprint in perpetuity. It is made available under aCC-BY-ND 4.0 International license.

\begin{tabular}{|c|c|c|c|c|c|c|c|}
\hline Symbol & Gene_name & AAgAtlas & \#Severe & \#Controls & \#Reactive_UCls & hits_FCs & Cluster_ID \\
\hline ASTL & astacin like metalloendopeptidase & no & 2 & 1 & 2 & $5.7,(3.8,7.5)$ & 1 \\
\hline BEND7 & BEN domain containing 7 & no & 6 & 1 & 7 & $5.5,(3.2,16.1)$ & 2 \\
\hline BLVRA & biliverdin reductase $A$ & no & 1 & 0 & 3 & $17.9,(17.9,17.9)$ & 1 \\
\hline BMPR2 & bone morphogenetic protein receptor type 2 & yes & 3 & 0 & 2 & $3.5,(3.2,4.0)$ & 1 \\
\hline C1orf94 & chromosome 1 open reading frame 94 & no & 12 & 0 & 8 & $5.2,(3.0,15.4)$ & 3 \\
\hline C3orf18 & chromosome 3 open reading frame 18 & no & 1 & 0 & 2 & 3.3, $(3.3,3.3)$ & 1 \\
\hline CALHM1 & calcium homeostasis modulator 1 & no & 3 & 1 & 2 & $3.9,(3.3,4.4)$ & 1 \\
\hline CAV2 & caveolin 2 & no & 9 & 0 & 2 & $3.7,(3.1,5.0)$ & 4 \\
\hline CCDC106 & coiled-coil domain containing 106 & no & 4 & 0 & 10 & $4.4,(3.1,7.2)$ & 2 \\
\hline CCDC146 & coiled-coil domain containing 146 & no & 5 & 0 & 3 & $3.6,(3.1,4.7)$ & 1 \\
\hline CD2BP2 & CD2 cytoplasmic tail binding protein 2 & no & 2 & 0 & 3 & $14.9,(5.1,24.8)$ & 5 \\
\hline CDC73 & cell division cycle 73 & no & 1 & 0 & 2 & $4.1,(4.1,4.1)$ & 1 \\
\hline CHMP7 & charged multivesicular body protein 7 & no & 10 & 1 & 3 & $3.6,(3.1,4.7)$ & 4 \\
\hline CTAG2 & cancer/testis antigen 2 & no & 3 & 0 & 6 & $5.2,(3.0,9.4)$ & 1 \\
\hline CYP2S1 & cytochrome P450 family 2 subfamily S member 1 & no & 2 & 0 & 3 & $4.1,(3.2,5.0)$ & 1 \\
\hline DNAJC17 & DnaJ heat shock protein family (Hsp40) member $\mathrm{C} 17$ & no & 4 & 0 & 2 & $3.2,(3.0,3.6)$ & 2 \\
\hline DOLPP1 & dolichyldiphosphatase 1 & no & 3 & 0 & 3 & $4.7,(3.9,5.1)$ & 1 \\
\hline EHD1 & EH domain containing 1 & no & 2 & 0 & 14 & $33.4,(3.6,63.2)$ & 1 \\
\hline EHD2 & EH domain containing 2 & no & 2 & 0 & 4 & $4.3,(3.0,5.6)$ & 1 \\
\hline ELOA2 & elongin A2 & no & 9 & 0 & 2 & $3.5,(3.0,4.9)$ & 4 \\
\hline EXD1 & exonuclease $3^{\prime}-5$ ' domain containing 1 & no & 1 & 0 & 2 & $7.2,(7.2,7.2)$ & 5 \\
\hline EXOC4 & exocyst complex component 4 & no & 17 & 0 & 7 & $3.9,(3.0,4.9)$ & 4 \\
\hline FAM185A & family with sequence similarity 185 member $A$ & no & 4 & 1 & 2 & $3.4,(3.2,3.6)$ & 1 \\
\hline FAM32A & family with sequence similarity 32 member $A$ & no & 4 & 0 & 2 & $3.6,(3.2,4.0)$ & 2 \\
\hline FBXL19 & F-box and leucine rich repeat protein 19 & no & 2 & 0 & 3 & $7.0,(3.0,11.0)$ & 1 \\
\hline FDFT1 & farnesyl-diphosphate farnesyltransferase 1 & no & 1 & 0 & 2 & $46.8,(46.8,46.8)$ & 1 \\
\hline FRG1 & FSHD region gene 1 & no & 5 & 0 & 3 & $3.6,(3.2,4.3)$ & 1 \\
\hline FUT9 & fucosyltransferase 9 & no & 2 & 1 & 3 & $3.9,(3.5,4.3)$ & 1 \\
\hline GATA2 & GATA binding protein 2 & no & 5 & 0 & 2 & $3.6,(3.0,4.3)$ & 4 \\
\hline GIMAP8 & GTPase, IMAP family member 8 & no & 1 & 0 & 2 & $4.7,(4.7,4.7)$ & 1 \\
\hline HNF4A & hepatocyte nuclear factor 4 alpha & no & 1 & 0 & 2 & $11.7,(11.7,11.7)$ & 1 \\
\hline HNRNPUL1 & heterogeneous nuclear ribonucleoprotein $\mathrm{U}$ like 1 & no & 3 & 0 & 4 & $5.7,(3.6,8.6)$ & 1 \\
\hline HPGD & 15-hydroxyprostaglandin dehydrogenase & no & 1 & 0 & 4 & $6.0,(6.0,6.0)$ & 1 \\
\hline IFNA10 & interferon alpha 10 & no & 2 & 0 & 5 & $18.8,(16.8,20.7)$ & 2 \\
\hline IFNA13 & interferon alpha 13 & no & 4 & 0 & 2 & $22.5,(4.6,51.4)$ & 2 \\
\hline IFNA14 & interferon alpha 14 & no & 3 & 0 & 2 & $19.3,(3.2,44.2)$ & 2 \\
\hline IFNA2 & interferon alpha 2 & yes & 2 & 0 & 3 & $42.5,(25.2,59.8)$ & 2 \\
\hline IFNA21 & interferon alpha 21 & no & 2 & 0 & 10 & $25.1,(14.9,35.3)$ & 2 \\
\hline IFNA5 & interferon alpha 5 & no & 2 & 0 & 3 & $14.6,(14.6,14.7)$ & 2 \\
\hline IFNA6 & interferon alpha 6 & no & 4 & 1 & 12 & $9.4,(3.3,21.8)$ & 2 \\
\hline IFNA8 & interferon alpha 8 & no & 7 & 0 & 5 & $9.7,(3.1,36.4)$ & 2 \\
\hline IFNL3 & interferon lambda 3 & no & 3 & 1 & 5 & $5.5,(4.2,7.6)$ & 1 \\
\hline IFNW1 & interferon omega 1 & no & 2 & 0 & 5 & $29.6,(10.6,48.5)$ & 2 \\
\hline IKZF3 & IKAROS family zinc finger 3 & no & 2 & 0 & 4 & $13.8,(3.3,24.2)$ & 1 \\
\hline KCNJ12 & potassium inwardly rectifying channel subfamily $\mathrm{J}$ member 12 & no & 1 & 0 & 2 & $3.1,(3.1,3.1)$ & 1 \\
\hline KCNJ14 & potassium inwardly rectifying channel subfamily $\mathrm{J}$ member 14 & no & 2 & 0 & 3 & $3.9,(3.2,4.6)$ & 1 \\
\hline KLHL31 & kelch like family member 31 & no & 1 & 0 & 2 & $11.3,(11.3,11.3)$ & 2 \\
\hline KLHL40 & kelch like family member 40 & no & 1 & 0 & 4 & $8.4,(8.4,8.4)$ & 2 \\
\hline LALBA & lactalbumin alpha & no & 1 & 0 & 2 & $3.9,(3.9,3.9)$ & 1 \\
\hline LINC01547 & long intergenic non-protein coding RNA 1547 & no & 2 & 0 & 6 & $19.3,(3.4,35.1)$ & 1 \\
\hline MAGEE1 & MAGE family member E1 & no & 1 & 0 & 3 & $17.9,(17.9,17.9)$ & 1 \\
\hline
\end{tabular}

Table S2. Proteins reactive in severe COVID-19 patients. Symbol, gene symbol. AAgAtlas, is protein listed in AAgAtlas 1.0? \#Severe, number of severe COVID-19 patients with reactivity to at least one UCI. \#Controls, number of control donors (healthy or mild-moderate COVID-19) with reactivity to at least one UCI. \#Reactive_UCIs, number of reactive UCIs associated with given ORF. Hits_FCs, mean and range (minimum to maximum) of per-ORF maximum hits fold-change observed among the patients with the reactivity. Cluster_ID, row cluster defined by Fig $\mathbf{4 B}$. 
bioRxiv preprint doi: https://doi.org/10.1101/2021.03.02.432977; this version posted March 3, 2021. The copyright holder for this preprint (which was not certified by peer review) is the author/funder, who has granted bioRxiv a license to display the preprint in perpetuity. It is made available under aCC-BY-ND 4.0 International license.

\begin{tabular}{|c|c|c|c|c|c|c|c|}
\hline Symbol & Gene_name & AAgAtlas & \#Severe & \#Controls & \#Reactive_UCls & hits_FCs & Cluster_ID \\
\hline MAX & MYC associated factor $\mathrm{X}$ & no & 7 & 0 & 10 & "13.0, (3.1,30.9) & 3 \\
\hline MBD3L1 & methyl-CpG binding domain protein 3 like 1 & no & 2 & 0 & 5 & $8.1,(4.1,12.2)$ & 1 \\
\hline MKX & mohawk homeobox & no & 6 & 1 & 3 & $3.8,(3.1,4.8)$ & 4 \\
\hline MPPED2 & metallophosphoesterase domain containing 2 & no & 5 & 0 & 3 & $5.2,(3.1,11.7)$ & 1 \\
\hline NACC1 & nucleus accumbens associated 1 & no & 2 & 0 & 12 & $74.9,(74.7,75.2)$ & 5 \\
\hline NAPSA & napsin $A$ aspartic peptidase & no & 3 & 1 & 3 & $4.1,(3.1,4.7)$ & 1 \\
\hline NBPF1 & NBPF member 1 & no & 1 & 0 & 2 & $6.9,(6.9,6.9)$ & 1 \\
\hline NBPF15 & NBPF member 15 & no & 1 & 0 & 2 & $3.5,(3.5,3.5)$ & 1 \\
\hline NOXO1 & NADPH oxidase organizer 1 & no & 3 & 0 & 6 & $3.9,(3.0,4.8)$ & 1 \\
\hline NT5C1A & 5'-nucleotidase, cytosolic IA & no & 3 & 1 & 2 & $26.9,(7.2,59.9)$ & 5 \\
\hline NUP62 & nucleoporin 62 & no & 1 & 0 & 7 & $8.4,(8.4,8.4)$ & 1 \\
\hline NVL & nuclear VCP like & no & 1 & 0 & 2 & $21.6,(21.6,21.6)$ & 1 \\
\hline OLFM4 & olfactomedin 4 & yes & 5 & 1 & 3 & $12.9,(4.4,29.8)$ & 5 \\
\hline PIMREG & PICALM interacting mitotic regulator & no & 4 & 1 & 4 & $3.8,(3.5,4.1)$ & 1 \\
\hline PLEKHF1 & pleckstrin homology and FYVE domain containing 1 & no & 2 & 0 & 3 & $3.3,(3.1,3.5)$ & 1 \\
\hline PML & PML nuclear body scaffold & no & 1 & 0 & 4 & $29.7,(29.7,29.7)$ & 1 \\
\hline PNMA1 & PNMA family member 1 & yes & 1 & 0 & 3 & $6.4,(6.4,6.4)$ & 2 \\
\hline PNMA5 & PNMA family member 5 & no & 2 & 0 & 5 & $5.7,(4.0,7.4)$ & 2 \\
\hline POLDIP3 & DNA polymerase delta interacting protein 3 & no & 5 & 0 & 3 & $3.3,(3.1,3.7)$ & 4 \\
\hline POMP & proteasome maturation protein & no & 1 & 0 & 2 & $3.2,(3.2,3.2)$ & 1 \\
\hline POU6F1 & POU class 6 homeobox 1 & no & 1 & 0 & 3 & $12.0,(12.0,12.0)$ & 1 \\
\hline PQBP1 & polyglutamine binding protein 1 & no & 5 & 0 & 2 & $3.2,(3.0,3.5)$ & 5 \\
\hline PRKAR2B & protein kinase cAMP-dependent type II regulatory subunit beta & no & 1 & 0 & 3 & $7.3,(7.3,7.3)$ & 1 \\
\hline PXDNL & peroxidasin like & no & 4 & 0 & 4 & $3.5,(3.1,3.9)$ & 2 \\
\hline RBM17 & RNA binding motif protein 17 & no & 1 & 0 & 3 & $23.6,(23.6,23.6)$ & 1 \\
\hline RCAN3 & RCAN family member 3 & no & 1 & 0 & 5 & $5.3,(5.3,5.3)$ & 1 \\
\hline RPL13AP3 & ribosomal protein L13a pseudogene 3 & no & 4 & 1 & 5 & $3.4,(3.1,3.7)$ & 1 \\
\hline RPL15 & ribosomal protein L15 & no & 11 & 1 & 6 & $3.4,(3.1,3.9)$ & 3 \\
\hline RPP14 & ribonuclease P/MRP subunit $\mathrm{p} 14$ & no & 1 & 0 & 6 & $35.9,(35.9,35.9)$ & 1 \\
\hline RPP30 & ribonuclease P/MRP subunit p30 & no & 1 & 0 & 4 & $46.1,(46.1,46.1)$ & 1 \\
\hline RUFY4 & RUN and FYVE domain containing 4 & no & 1 & 0 & 4 & $16.3,(16.3,16.3)$ & 1 \\
\hline SNRPA1 & small nuclear ribonucleoprotein polypeptide $A^{\prime}$ & no & 1 & 0 & 2 & $5.3,(5.3,5.3)$ & 1 \\
\hline SPEF1 & sperm flagellar 1 & no & 2 & 0 & 5 & $5.9,(3.2,8.5)$ & 1 \\
\hline SPRR1B & small proline rich protein $1 \mathrm{~B}$ & no & 1 & 0 & 4 & $7.5,(7.5,7.5)$ & 1 \\
\hline SSNA1 & SS nuclear autoantigen 1 & yes & 1 & 0 & 5 & $12.2,(12.2,12.2)$ & 2 \\
\hline STPG3 & sperm-tail PG-rich repeat containing 3 & no & 1 & 0 & 2 & $3.6,(3.6,3.6)$ & 1 \\
\hline SYT2 & synaptotagmin 2 & no & 6 & 1 & 4 & $3.6,(3.2,4.5)$ & 5 \\
\hline TBC1D10B & TBC1 domain family member $10 \mathrm{~B}$ & no & 3 & 1 & 2 & $3.4,(3.1,4.1)$ & 1 \\
\hline TFAP4 & transcription factor AP-4 & no & 1 & 0 & 5 & $3.7,(3.7,3.7)$ & 1 \\
\hline TMPO & thymopoietin & no & 2 & 0 & 5 & $15.7,(3.7,27.7)$ & 1 \\
\hline TNFSF14 & TNF superfamily member 14 & no & 1 & 0 & 2 & $3.7,(3.7,3.7)$ & 1 \\
\hline TOX4 & TOX high mobility group box family member 4 & no & 1 & 0 & 3 & $9.9,(9.9,9.9)$ & 1 \\
\hline TRAIP & TRAF interacting protein & no & 2 & 0 & 3 & $4.7,(3.4,6.0)$ & 1 \\
\hline VAV1 & vav guanine nucleotide exchange factor 1 & no & 1 & 0 & 4 & $10.2,(10.2,10.2)$ & 1 \\
\hline ZBTB18 & zinc finger and BTB domain containing 18 & no & 1 & 0 & 2 & $3.3,(3.3,3.3)$ & 1 \\
\hline ZFP2 & ZFP2 zinc finger protein & no & 2 & 1 & 3 & $4.9,(3.1,6.7)$ & 1 \\
\hline ZMAT2 & zinc finger matrin-type 2 & no & 6 & 0 & 5 & $3.6,(3.1,4.1)$ & 2 \\
\hline ZNF146 & zinc finger protein 146 & no & 2 & 0 & 8 & $16.4,(3.1,29.7)$ & 1 \\
\hline ZNF232 & zinc finger protein 232 & no & 2 & 1 & 4 & $4.6,(3.5,5.8)$ & 1 \\
\hline ZNF678 & zinc finger protein 678 & no & 3 & 1 & 2 & $6.6,(3.4,12.8)$ & 1 \\
\hline ZSCAN32 & zinc finger and SCAN domain containing 32 & no & 1 & 0 & 2 & $11.3,(11.3,11.3)$ & 1 \\
\hline ZSCAN5A & zinc finger and SCAN domain containing $5 \mathrm{~A}$ & no & 3 & 0 & 4 & $4.0,(3.2,5.3)$ & 1 \\
\hline
\end{tabular}

Table S2 (continued). Proteins reactive in severe COVID-19 patients. 
bioRxiv preprint doi: https://doi.org/10.1101/2021.03.02 432977; this version posted March 3, 2021. The copyright holder for this preprint (which was not certified by peer review) is the author/funder, who has granted bioRxiv a license to display the preprint in perpetuity. It is made available under aCC-BY-ND 4.0 International license.

\begin{tabular}{|c|c|}
\hline Names & Sequences \\
\hline T7-Pep2_PCR1_F & 5'-ATAAAGGTGAGGGTAATGTC-3' \\
\hline Nextera Index 1 & 5'-CAAGCAGAAGACGGCATACGAGAT[i7]GTCTCGTGGGCTCGG-3' \\
\hline PhIP_PCR2_F & 5'-AATGATACGGCGACCACCGAGATCTACAC[i5]GGAGCTGTCGTATTCCAGTC-3' \\
\hline P7.2 & 5'-CAAGCAGAAGACGGCATACGA-3' \\
\hline T7-Pep2_PCR1_R+ad_min & 5'-CTGGAGTTCAGACGTGTGCTCTTCCGATCAGTTACTCGAGCTTATCGT-3' \\
\hline Ad_min_BCX_P7 & 5'-CAAGCAGAAGACGGCATACGAGAT[i7]CTGGAGTTCAGACGT-3' \\
\hline T7-Pep2.2_SP_subA & 5'-CTCGGGGATCCAGGAATTCCGCTGCGT-3' \\
\hline MISEQ_MIPSA_R2 & 5'- ATGACGACAAGCCATGGTCGAATCAAACAAGTTTGTACAAAAAAGTTGGC-3' \\
\hline MIPSA_i5_NextSeq_SP & 5'-GGATCCCCGAGACTGGAATACGACAGCTCC-3' \\
\hline Standard_i7_SP & 5'-GATCGGAAGAGCACACGTCTGAACTCCAGTCAC-3' \\
\hline HL-32_ad & HL-GACGTGTGCTCTTCCGATCAAATTATTTCTAGGTACTCGAGCTTATCG-3' \\
\hline MX1_Forward & 5'-ACCACAGAGGCTCTCAGCAT-3' \\
\hline MX1_Reverse & 5'-CTCAGCTGGTCCTGGATCTC-3' \\
\hline GAPDH_Forward & 5'-GAGTCAACGGATTTGGTCGT-3' \\
\hline GAPDH_Reverse & 5'-TTGATTTTGGAGGGATCTCG-3' \\
\hline BT2_F & 5'-GTCAGAGTGACACACTGT-3' \\
\hline BT2_R & 5'-AGAGTGACAGTCACAGTG-3' \\
\hline BG4_F & 5'-CACTGACTGTGTGAGTGT-3' \\
\hline BG4_R & 5'-TGAGACACAGTGAGTCAC-3' \\
\hline NT5C1A_F & 5'-CTCACAGACAGACGTCA-3' \\
\hline NT5C1A_R & 5'-TGTCAGTCAGTGAGTGTG-3' \\
\hline
\end{tabular}

Table S3. Primer sequences used in this study.

Data S1. Hits fold-change MIPSA data matrix for UCIs of reactive proteins in severe

COVID-19 patients.

(separate file) 